\title{
Constraining supermassive black hole evolution through the continuity equation
}

\author{
Marco Tucci ${ }^{1}$ and Marta Volonteri ${ }^{2}$ \\ 1 Département de Physique Théorique and Center for Astroparticle Physics, Université de Genève, 24 quai Ansermet, 1211 Genève 4 , \\ Switzerland \\ e-mail: Marco.Tucci@unige.ch \\ 2 Institut d'Astrophysique de Paris, Sorbonne Universitès, UPMC Univ. Paris 6 et CNRS, UMR 7095, 98 bis bd Arago, 75014 Paris, \\ France
}

Received 2 March 2016 / Accepted 12 January 2017

\begin{abstract}
The population of supermassive black holes (SMBHs) is split between those that are quiescent, such as those seen in local galaxies including the Milky Way, and those that are active, resulting in quasars and active galactic nuclei (AGN). Outside our neighborhood, all the information we have on SMBHs is derived from quasars and AGN, giving us a partial view. We study the evolution of the SMBH population, total and active, by the continuity equation, backwards in time from $z=0$ to $z=4$. Type- 1 and type-2 AGN are differentiated in our model on the basis of their respective Eddington ratio distributions, chosen on the basis of observational estimates. The duty cycle is obtained by matching the luminosity function of quasars, and the average radiative efficiency is the only free parameter in the model. For higher radiative efficiencies $(\gtrsim 0.07)$, a large fraction of the SMBH population, most of them quiescent, must already be in place by $z=4$. For lower radiative efficiencies $(\sim 0.05)$, the duty cycle increases with the redshift and the SMBH population evolves dramatically from $z=4$ onwards. The mass function of active SMBHs does not depend on the choice of the radiative efficiency or of the local SMBH mass function, but it is mainly determined by the quasar luminosity function once the Eddington ratio distribution is fixed. Only direct measurement of the total black-hole mass function at redshifts $z \gtrsim 2$ could break these degeneracies, offering important constraints on the average radiative efficiency. Focusing on type-1 AGN, for which observational estimates of the mass function and Eddington ratio distribution exist at various redshifts, models with lower radiative efficiencies better reproduce the high-mass end of the mass function at high $z$, but tend to over-predict it at low $z$, and vice-versa for models with higher radiative efficiencies.
\end{abstract}

Key words. galaxies: active - galaxies: evolution - galaxies: luminosity function, mass function - quasars: supermassive black holes

\section{Introduction}

The population of supermassive black holes (SMBHs) we detect at the center of local galaxies (e.g., Kormendy \& Richstone 1995) has been built over long cosmic times, as traced, for instance, by the luminosity function of active galactic nuclei (AGN) and quasars. Local SMBHs represent the relics of the young population observed at earlier cosmic epochs.

While at $z=0$, for at least several tens of galaxies, we can measure the SMBH mass (for a review of the masses and the measurement techniques, we refer to Kormendy \& Ho 2013); this is impossible at higher redshift, where we can only obtain estimates of SMBH masses through indirect methods. In fact, as soon as we move away from the local Universe, we can only observe active SMBHs, powering AGN and quasars, giving us only a partial view of the full population. For each AGN, there may be several dormant SMBHs that we cannot identify as such.

There are different ways of theoretically estimating the cosmic evolution of the SMBH population. Analytical models, for instance, can use the continuity equation (see, e.g., Cavaliere et al. 1971; Small \& Blandford 1992; Yu \& Tremaine 2002; Marconi et al. 2004; Merloni \& Heinz 2008; Shankar et al. 2013), which relies on the assumption that the mass function of
SMBHs at one time can be evolved in time, backwards or forwards, to predict the mass function at an earlier or later time, depending on the distribution of accretion rates. This kind of models assumes that SMBHs grow primarily by gas accretion in luminous phases, and that the luminosity function of AGN can be used as a constraint. Another option is to convolve the dark matter halo mass function and/or merger rate with a relation between SMBH and halo, and track the SMBHs by assuming that the link with the properties of dark matter halos is sufficient to describe the main properties of the SMBH population (e.g., Haiman \& Loeb 1998; Wyithe \& Loeb 2002, 2003).

Alternatively, one can use semi-analytical models, where the population of SMBHs is evolved jointly with the dark matter halos and galaxies hosting them, using analytical prescription to describe the main physical processes such as gas cooling, star-formation rate, and accretion onto the SMBHs (e.g., Kauffmann \& Haehnelt 2000; Cattaneo 2001; Volonteri et al. 2003; Monaco et al. 2000, 2007; Haehnelt \& Kauffmann 2000; Cattaneo et al. 2005; Croton et al. 2006; Bower et al. 2006; Fontanot et al. 2011; Hirschmann et al. 2012). The main assumption in this case is that the properties of dark matter halos determine those of the galaxies and SMBHs, 
and that baryonic physics can be described with analytical expressions.

Finally, one can use cosmological hydrodynamical simulations (e.g., Sijacki et al. 2007; Di Matteo et al. 2008; Booth \& Schaye 2009; Dubois et al. 2010, 2012; Hirschmann et al. 2014; Sijacki et al. 2015; Volonteri et al. 2016), where the advantage is that the cosmological environment is followed faithfully in the non-linear regime, and the growth of galaxies through mergers and gas accretion is naturally taken into account. However, small-scale baryonic physics is included using analytical approximations as in the case of semi-analytical models, and such simulations are computationally expensive, excluding the possibility of exploring a large parameter space, as is instead possible using analytical or semi-analytical techniques.

This paper is the first in a series of two, where we first develop a framework based on the continuity equation to follow the cosmic evolution of SMBHs (this paper) and we then apply this framework to model the AGN luminosity function at radio wavelengths, where little dedicated work has thus far been done (e.g., Haiman et al. 2004; Shankar et al. 2010).

The outline of the paper is as follows. In Sect. 2, we recall the main properties of the continuity equation and its implementation. In Sect. 3, we describe our methodology and assumptions. In Sect. 4, we present the main results and their discussion, and, finally, in Sect. 5, we summarize our conclusions.

\section{SMBH evolution via a continuity equation}

The evolution of the SMBH population through time is typically described by a continuity equation (see, e.g., Cavaliere et al. 1971; Small \& Blandford 1992; Yu \& Tremaine 2002; Marconi et al. 2004; Merloni \& Heinz 2008; Shankar et al. 2013):

$$
\frac{\partial \Phi_{\mathrm{BH}}}{\partial t}(M, t)=-M \frac{\partial}{\partial M}\left[\frac{\langle\dot{M}\rangle(M, t) \Phi_{\mathrm{BH}}(M, t)}{M}\right],
$$

where $\Phi_{\mathrm{BH}}(M, t)$ (hereafter, we use the symbol $\Phi(x)$ to denote functions in logarithmic units, that is, $\Phi(x) \equiv \mathrm{d} N / \mathrm{d} \log (x))$ is the black-hole mass function (BHMF), defined as the number of SMBHs per co-moving volume with mass in the logarithmic interval $\log (M), \log (M)+\Delta \log (M)$ and $\langle\dot{M}\rangle$ is the average accretion rate of SMBHs of mass $M$ at time $t$. Under the assumption that SMBHs grow during phases of AGN activity, the growth rate is the result of the mass that falls into the black hole but is not converted into energy. If $\dot{M}_{\text {acc }}$ is the accretion rate of matter onto a $\mathrm{SMBH}$, the part of it converted into luminosity is $L=\epsilon \dot{M}_{\text {acc }} c^{2}$, where $\epsilon$ is the radiative efficiency of the accretion flow. The growth rate of a SMBH is thus given by $\dot{M}=(1-\epsilon) \dot{M}_{\text {acc }}$. Consequently, the bolometric luminosity can be related to the mass accretion rate by

$L=\frac{\epsilon}{(1-\epsilon)} c^{2} \dot{M}(M, t)$

Although individual SMBHs turn on and off, the mass function evolution depends only on the average accretion rate of all SMBHs, active and inactive. In terms of the Eddington ratio, defined as $\lambda \equiv L / L_{\text {Edd }}$ with the Eddington luminosity $L_{\text {Edd }}=\ell M$ and $\ell \simeq 1.26 \times 10^{38} M_{\odot}^{-1} \mathrm{erg} \mathrm{s}^{-1}$, the average accretion rate is

$\frac{\langle\dot{M}\rangle(M, t)}{M}=\frac{(1-\epsilon) \ell}{\epsilon c^{2}} U(M, t)\langle\lambda\rangle(M, t)$, where $\epsilon$ now has to be considered as the average value for SMBHs of mass $M$ at time $t$. The average Eddington ratio $\langle\lambda\rangle$ is

$\langle\lambda\rangle(M, t)=\int \operatorname{dlog} \lambda P(\lambda \mid M, t) \lambda$,

where $P(\lambda \mid M, t)$ is the Eddington ratio distribution, that is, the probability for a SMBH of mass $M$ to accrete at the Eddington ratio $\lambda$ at time $t$ (it is per unit of $\log \lambda$ and is normalized to unity, i.e., $\left.\int \mathrm{d} \log \lambda P(\lambda \mid M, t)=1\right)$.

The average accretion rate also depends on the duty cycle, $U(M, t)$, that is, on the fraction of SMBHs of mass $M$ that are active at time $t$. By the definition of duty cycle, we can introduce the mass function of active SMBHs (throughout the paper, this is also referred to as the AGN MF):

$\Phi_{\mathrm{AGN}}(M, t)=\Phi_{\mathrm{BH}}(M, t) U(M, t)$.

Using Eqs. (3) and (5), the continuity equation can be written as

$$
\frac{\partial \Phi_{\mathrm{BH}}}{\partial z}=-\frac{\ell}{c^{2} \ln (10)} \frac{\mathrm{d} t}{\mathrm{~d} z} \frac{\partial}{\partial \log M}\left[\frac{(1-\epsilon)}{\epsilon}\langle\lambda\rangle \Phi_{\mathrm{AGN}}\right] .
$$

The AGN MF can be also related to the bolometric luminosity function of quasars by the following equation

$\Phi(L, z)=\int \operatorname{dlog} \lambda P(\lambda \mid M, z) \Phi_{\mathrm{AGN}}(M, z)$.

The quasar luminosity function is usually taken as input in models based on the continuity equation, and used to constrain the evolution of the AGN MF.

Finally, we note that the contribution from black hole mergers has not been included in Eq. (1). The relevance of this term is relatively unknown and is difficult to evaluate. Shankar et al. (2013) attempted to estimate this effect using the SMBH merger rate predicted by hierarchical models of structure formation. They found that mergers have limited effects at $z>2$, but that they might be relevant on the local mass function increasing the space density of high-mass SMBHs (see also the discussion in Merloni \& Heinz 2008). Aversa et al. (2015) agree with these conclusions. They found that mergers moderately increase the space densities of SMBHs with $M \gtrsim 10^{9} M_{\odot}$ at low redshifts, but that the effect is probably smaller than the current uncertainties on the local BHMF. Numerical simulations by Volonteri et al. (2005), Berti \& Volonteri (2008) also verified that mass accretion dominates over mergers in determining the mass growth and spin distribution of black holes. Because of the large uncertainties in both merging rates of galaxies and physical processes involved in black hole mergers, we neglect this contribution in the following analysis. This is a standard assumption in most of the studies that employ the continuity equation.

\section{Method}

Below, we describe the method we employ to solve the continuity equation (Eq. (6)). We use the local BHMF as boundary condition and integrate the equation backwards in time up to redshift $z=4$. The continuity equation also requires the simultaneous knowledge of the Eddington ratio distribution, the duty cycle, and the average radiative efficiency of SMBHs.

Briefly, the inputs of the model are the following:

- Local BHMF. We use two possible mass functions, based on results of Shankar et al. (2009) and Shankar (2013). 
- Eddington ratio distribution. We consider different distributions for type-1 and type-2 AGN on the basis of the observational estimates of Kelly \& Shen (2013) and Aird et al. (2012).

- Average radiative efficiency $\epsilon$. This is a free parameter of the model. We study solutions of the continuity equation for different values of $\epsilon$, assuming it to be independent of time and of SMBH mass.

- Quasar luminosity function $(Q L F)$. We adopt the observational QLF from Hopkins et al. (2007).

Finally, the duty cycle is described by a parametric function (double power law) of redshift and SMBH mass. The parameters are determined as the best fit of the observational quasar luminosity function, employing a Markov chain Monte Carlo (MCMC) method. Jointly with the BHMF, the duty cycle is then the main output of the model.

In the following, we detail the main ingredients and assumptions of the model.

\subsection{Local BHMF}

Several works estimated the local BHMF using the observed empirical scaling relations between SMBH mass and host galaxy spheroidal properties, such as luminosity, stellar velocity dispersion, and bulge mass (see Shankar et al. 2009; Kormendy \& Ho 2013, for a review). Shankar et al. (2009) present a compilation of local BHMF determinations based on a variety of methods, scaling relations, and data sets. The spread in the estimates is shown in Fig. 1 (the blue shaded area). These estimates give a local mass density of SMBHs in the range $\rho_{\mathrm{BH}}=3.2-$ $5.4 \times 10^{5} M_{\odot} \mathrm{Mpc}^{-3}$. Moreover, Vika et al. (2009) derive the local BHMF for a large sample of galaxies from the Millennium Galaxy Catalogue using the empirical black hole mass-bulge luminosity relation of Graham (2007). Li et al. (2011) use luminosity and stellar mass functions of field galaxies to constrain the masses of their spheroids, and then compute SMBH mass through the empirical correlation between SMBH and spheroid mass (Häring \& Rix 2004). Despite the different methods, all these derived local BHMFs are consistent with one another (see Fig. 1).

However, uncertainties on the actual local BHMF do remain. The main issue is related to the scaling relationships used for the SMBH mass determination. Recently, Kormendy \& Ho (2013) have updated the calibration between the SMBH mass and the luminosity, mass, or velocity dispersion of the bulge component of the host galaxy in the local Universe. This led to an upward revision by a factor of approximately 2-3 of previously computed SMBH masses. Assuming the new relation of Kormendy \& Ho (2013), Ueda et al. (2014) updated the local mass function of Li et al. (2011) finding a larger MF at all masses. Moreover, Shankar (2013) derived the local BHMF from the assumption that all local galaxies follow the revised early-type $M_{\mathrm{BH}}-\sigma$ relation from McConnell \& Ma (2013). In this case, the revised estimates give a significantly larger local MF at the highest masses $\left(\gtrsim 10^{9} M_{\odot}\right)$ with respect to previous computations (see Fig. 1).

Following Merloni \& Heinz (2008) we adopt an analytic expression for the local BHMF, given by the convolution of a Schechter function with a Gaussian scatter. The Schechter function has the following parametrization:

$\Psi_{M}=\phi_{\star}\left(\frac{M}{M_{\star}}\right)^{1+\alpha} \exp \left(1-\frac{M}{M_{\star}}\right)$,

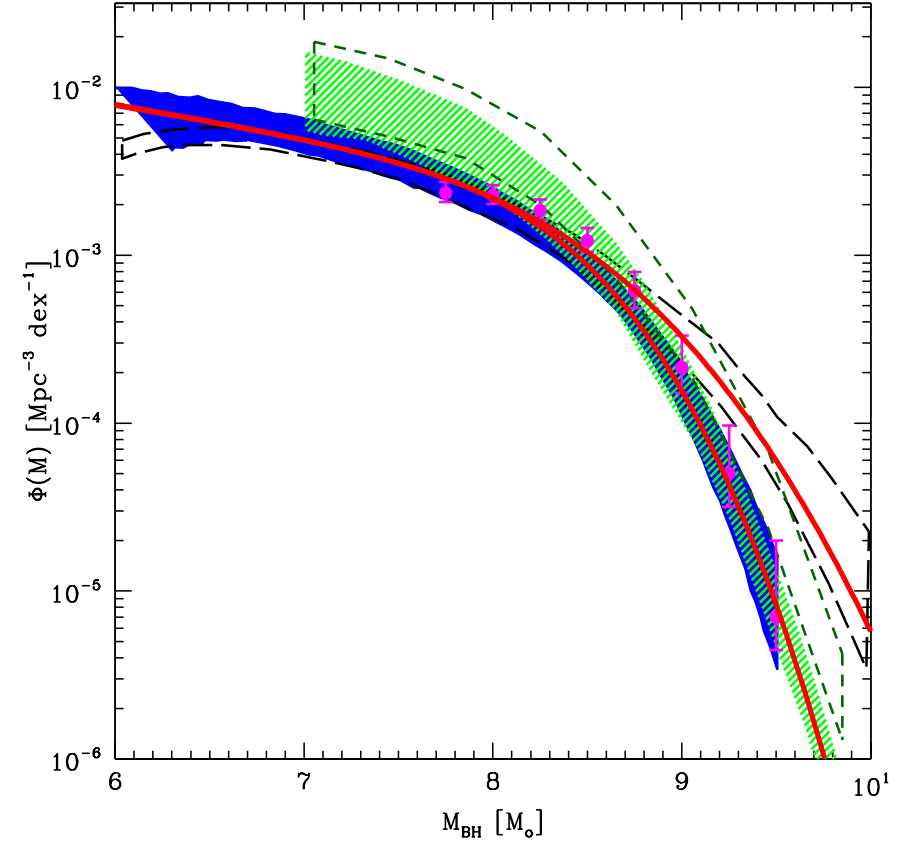

Fig. 1. Local BHMF as estimated by Shankar et al. (2009, blue shaded area), Vika et al. (2009, magenta points), Li et al. (2011, green lined area), Shankar (2013, black dashed region), and Ueda et al. (2014, dark green short-dashed region). Red solid thick lines correspond to the Schecter function convolved with Gaussian scatter of 0.3 dex (lower line) and $0.5 \mathrm{dex}$ (upper line).

with $\phi_{\star}=10^{-3}, \log M_{\star}=8.4$, and $\alpha=-1.19$. These parameters are chosen in order to give a mass function that is coincident with the central value within the uncertainty range of Shankar et al. (2009) if a Gaussian scatter of 0.3 dex is used.

In the following analysis, we use a local BHMF computed with a Gaussian scatter of 0.3 and $0.5 \mathrm{dex}$. In this way, we can take into account the current uncertainties on the local BHMF at the highest masses. Increasing the scatter from 0.3 to 0.5 dex, the mass function becomes compatible with estimates of Shankar (2013): the number density of SMBHs is larger by a factor $\sim 2$ at $M \sim 10^{9} M_{\odot}$, and by an order of magnitude at $M \sim 6 \times 10^{9} M_{\odot}$. Changing the scatter has only, however, a moderate impact on the local SMBH mass density: $\rho_{\mathrm{BH}}=4.3(6.6) \times 10^{5} M_{\odot} \mathrm{Mpc}^{-3}$ for a Gaussian scatter of $0.3(0.5)$ dex.

\subsection{Radiative efficiency}

The average radiative efficiency $\epsilon$ of quasars is commonly estimated based on the classical "Soltan argument" (Soltan 1982), according to which the local mass budget of black holes in galactic nuclei should be accounted for by integrating the overall energy density released by AGN with an appropriate mass-toenergy conversion efficiency. Several studies, assuming a fixed radiative efficiency over black hole mass and redshift, concluded that $\epsilon$ has to be within the range $0.05-0.40$ to explain the relic population (e.g., Yu \& Tremaine 2002; Elvis et al. 2002; Marconi et al. 2004; Shankar et al. 2009; Li et al. 2012). The same range of values is also predicted by the standard accretion disc theory (Shakura \& Sunyaev 1973), from $\epsilon=0.054$ for a non-rotating $\mathrm{SMBH}$ to $\epsilon=0.42$ for a SMBH with maximal spin.

Individual AGN can also be used to deduce the absolute accretion rate and the radiative efficiency by fitting observed optical spectra with the thin accretion disk model (Davis \& Laor 2011; Wu et al. 2013). Average values of $\epsilon \approx 0.1$ are derived, 
approximately consistent with estimates based on the Soltan argument.

It is still debated whether or not the radiative efficiency depends on SMBH mass and redshift. Theoretical arguments have suggested that the radiative efficiency may increase with the SMBH mass (e.g., Volonteri et al. 2007; Fanidakis et al. 2011). Davis \& Laor (2011) directly determined the radiative efficiency for 80 quasars and found a strong correlation with the SMBH mass. On the other hand, Wu et al. (2013) showed that the SDSS quasar data are consistent with no intrinsic correlation with the SMBH mass and no redshift evolution. They also demonstrated that the apparent correlation $\epsilon-M$ can be produced by selection effects and bias induced by mass estimates (see also Raimundo et al. 2012). Results based on Soltan's argument are also contrasting: Cao \& Li (2008), Wang et al. (2009), Li et al. (2012) are in favor of a mass and redshift dependence, while Cao (2010), Shankar et al. (2013) reach opposite conclusions.

For simplicity, our analysis assumes that $\epsilon$ is constant. We solve the continuity equation for different values of $\epsilon$, mainly between 0.05 and 0.1 (larger values of $\epsilon$ give small or negligible evolution of the BHMF in time, and they are discussed in Appendix A).

\subsection{Active SMBHs}

Throughout the paper, we define an active SMBH (or, equivalently, an AGN) if the Eddington ratio is $\lambda \geq \lambda_{\text {cut }}=10^{-4}$ At lower Eddington ratios, SMBHs are supposed to be quiescent. This choice is somewhat arbitrary if the Eddington ratio distribution is broad and extends to $\lambda<10^{-4}$ as indicated by different observations (e.g., Panessa et al. 2006; Hopkins et al. 2006; Babić et al. 2007). We choose this $\lambda_{\text {cut }}$ because SMBHs with smaller Eddington ratios do not give relevant contributions to the bolometric luminosity function, at least in the luminosity range we consider, that is, $10^{43}-10^{48} \mathrm{erg} \mathrm{s}^{-1}$. In fact, if $\lambda<10^{-4}$, only SMBHs with mass $M \gtrsim 10^{9} M_{\odot}$ have luminosity $L \geq 10^{43} \mathrm{erg} \mathrm{s}^{-1}$. The number density of SMBHs exponentially declines at these masses, that is, much faster than the power-law increase of the Eddington ratio distribution at low $\lambda$ as seen, for example, by Aird et al. (2012).

The duty cycle is clearly sensitive to the Eddington ratio limit defining an active SMBH. A lower threshold obviously increases the fraction of active SMBHs, but, in principle, the BHMF determined by the continuity equation should be independent of this. However, the evolution of the BHMF is affected by $\lambda_{\text {cut }}$ through the average accretion rate that is proportional to the product of the duty cycle and $\langle\lambda\rangle$ (see Eq. (3)). As shown in Appendix A, $\lambda_{\text {cut }}$ seems to not be particularly relevant for the mass function of both total and active SMBHs. Moreover, the choice of $\lambda_{\text {cut }}$ is still less important when we compare the model predictions with estimates from observational samples in which quasars are selected above a given flux limit and that are sensitive only to a specific range of Eddington ratios.

An alternative view, considered, for example, by Merloni \& Heinz (2008), assumes that every SMBH is active at some level, and that there is no distinction between SMBHs and AGN. By definition, the duty cycle is then equal to 1 . However, this implies the knowledge of the Eddington ratio distribution at very low accretion rates, $\lambda \ll 10^{-4}$, which are scarcely accessible to observations.

\subsection{Eddington ratio distribution}

There are observational evidences that the Eddington ratio distribution is different for type-1 (or unobscured) and type-2 (or obscured) AGN. Trump et al. (2011) proposed two distinct distributions of Eddington ratios for X-ray-selected AGN, corresponding to two different modes of accretion. They found that, in general, broad emission lines are present only at high accretion rates $\left(\lambda>10^{-2}\right)$. According to this study, unobscured AGN (and possibly some obscured narrow-line AGN) should be fed by a thin accretion disk containing the broad-line region and some obscuring material. On the other hand, narrow-line or lineless AGN at $\lambda<10^{-2}$ may be powered by a geometrically thick, radiatively inefficient accretion flow. Lusso et al. (2012) studied the Eddington ratio distribution for an X-ray sample of type-1 and type-2 AGN covering redshifts $z \leq 2$. The Eddington ratio distribution for type-1 AGN was found to be more consistent with a Gaussian than a power law. For type-2 AGN, results are less clear, with some evidence for a power-law distribution only at low redshifts. The presence of two distinct regimes for the SMBH growth was also pointed out by Kauffmann \& Heckman (2009). They analyzed the observed distribution of Eddington ratios for a sample of nearby SDSS galaxies. They found that galaxies with significant star formation are characterized by a broad log-normal distribution of accretion rates peaked at a few percent of the Eddington limit. On the contrary, galaxies with old central stellar populations are characterized by a power-law distribution function of Eddington ratios.

The Eddington ratio distribution for type-1 AGN was accurately determined by Shen \& Kelly (2012), Kelly \& Shen (2013) in a large redshift range, from 0.3 to 5 . They jointly estimated the BHMF and the Eddington ratio distribution function (ERDF) for a sample of approximately 58000 type-1 quasars from the SDSS. They employed a Bayesian technique to deal with selection effects and the statistical scatter between true SMBH masses and virial mass estimates. In particular, Kelly \& Shen (2013) modeled the joint bivariate distribution of SMBH mass and Eddington ratio as a superposition of 2D log-normal functions. Their results on the ERDF at different redshift bins show that the co-moving number densities of type- 1 quasars increase toward lower Eddington ratios, a trend that continues beyond the incompleteness limit. The only exceptions are the $z=3.75$ and $z=4.25$ bins, which display evidence for a peak at $\lambda \approx 0.3$. The distribution of $\lambda$ is relatively independent of the black hole mass at both low $(z \lesssim 0.6)$ and high $(z \gtrsim 3.2)$ redshift, while at intermediate redshifts, they found larger Eddington ratios moving from $M \sim 5 \times 10^{8} M_{\odot}$ to $M \sim 5 \times 10^{9} M_{\odot}$. However, as they commented, the mass dependence could not be real but be driven by systematic effects related to the change of the mass estimator in these redshift bins.

Concerning type-2 AGN, different independent works agree that the Eddington ratio distribution has a power law behavior. Hopkins \& Hernquist (2009) showed that the observed distributions from the complete sample of SDSS galaxies selected by Heckman et al. (2004), Yu et al. (2005) are well fitted by a Schecter function with a power law slope of approximately -0.6 , almost independent of the mass range, and a cut-off at $\lambda \sim 1$. Aird et al. (2012) estimated the distribution of the specific accretion rate, the rate of black hole growth relative to the stellar mass of the host galaxy, for a large sample of obscured X-ray AGN at redshift $0.2<z<1.0$. They found that the distribution is independent of the stellar mass of the host galaxy and can be described by a power law with constant slope $\simeq-0.65$ throughout the specific accretion rate $\left(10^{-4}-1\right)$ 
and redshift range. These results can be interpreted in terms of Eddington ratio assuming a direct proportionality between the mass of the central SMBH and the stellar mass of the host galaxy (they consider $M_{\mathrm{BH}} \approx 0.002 M_{\star}$ ). Similar results were also obtained by Bongiorno et al. (2012) from an X-ray and optically selected sample of AGN, but with a slightly steeper power-law index $(\approx-1)$.

Based on these observational findings, we model the Eddington ratio distribution, $P(\lambda)$, for type- 1 and type- 2 AGN separately. In both cases, we assume distributions independent of the SMBH mass. According to our definition of active SMBHs, $P(\lambda)$ is only considered for $\lambda \geq 10^{-4}$.

For type-1 AGN, we use a log-normal distribution,

$P_{1}(\lambda, z)=\frac{1}{2 \pi \sigma(z) \lambda} \mathrm{e}^{-\left[\ln \lambda-\ln \lambda_{c}(z)\right]^{2} / 2 \sigma^{2}(z)}$,

where the central value $\lambda_{\mathrm{c}}(z)$ and the dispersion $\sigma(z)$ of the distribution are determined by fitting the shape of the ERDFs from Kelly \& Shen (2013) in the different redshift bins and by interpolating the results with a linear function (see Fig. 2). We find

$\log \lambda_{\mathrm{c}}(z)=\max [-1.9+0.45 z, \log (0.03)]$

$\sigma(z)=\max (1.03-0.15 z, 0.6)$.

For type-2 AGN, we use a power-law distribution with an exponential cut-off at super-Eddington luminosities,

$P_{2}(\lambda, z)=a_{2}(z) \lambda^{\alpha_{\lambda}(z)} \mathrm{e}^{-\lambda / \lambda_{0}}$,

where $\lambda_{0}=1.5$ (or 2.5 when $\epsilon \geq 0.1$ ). The distribution is normalized to unity by the factor $a_{2}$. The slope of the power law is taken to be

$\alpha_{\lambda}= \begin{cases}-0.6 & z \leq 0.6 \\ -0.6 /(0.4+z) & z>0.6 .\end{cases}$

At low redshifts, this is in agreement with findings from Hopkins \& Hernquist (2009), Kauffmann \& Heckman (2009), Aird et al. (2012). At redshifts $z \gtrsim 1$, there are no observational constraints on the Eddington ratio distribution for type-2 AGN. Here we introduce a redshift dependence in $\alpha_{\lambda}$ that makes the distribution flatter and flatter at high redshifts. As discussed in Appendix A, keeping a constant slope of -0.6 would give a BHMF that does not significantly evolve over time at $z \gtrsim 0.5$ and at low and intermediate masses, in contrast with general expectations. For example, Fig. 3 shows the evolution of the BHMF computed from the continuity equation, assuming $U(M, t)=1$ and a powerlaw Eddington ratio distribution with constant slope $\alpha_{\lambda}=-0.6$ and -0.3 . Even with a duty cycle equal to 1 , most of SMBHs with $M<10^{8} M_{\odot}$ should already be formed before $z=4$ if $\alpha_{\lambda}=-0.6$. To be noted, the large difference in the mass function evolution between the two values of $\alpha_{\lambda}$.

As explained in Hopkins \& Hernquist (2009), given the quasar luminosity function, the Eddington ratio distribution can be directly translated into a quasar lifetime or light curve model. A log-normal distribution, as we use for type-1 AGN, is typically associated to "light bulb" models, in which quasars grow at fixed Eddington ratio with an instantaneous or exponential luminosity decay. A truncated power-law Eddington distribution arises instead if the quasar luminosity undergoes a power-law decay with time. Our assumptions on $P(\lambda)$ imply therefore a different accretion model for type- 1 and type- 2 AGN.

The Eddington ratio distribution for all the active AGN is thus the sum of the distributions for type- 1 and type- 2 quasars weighted by the relative abundance of the two populations. The

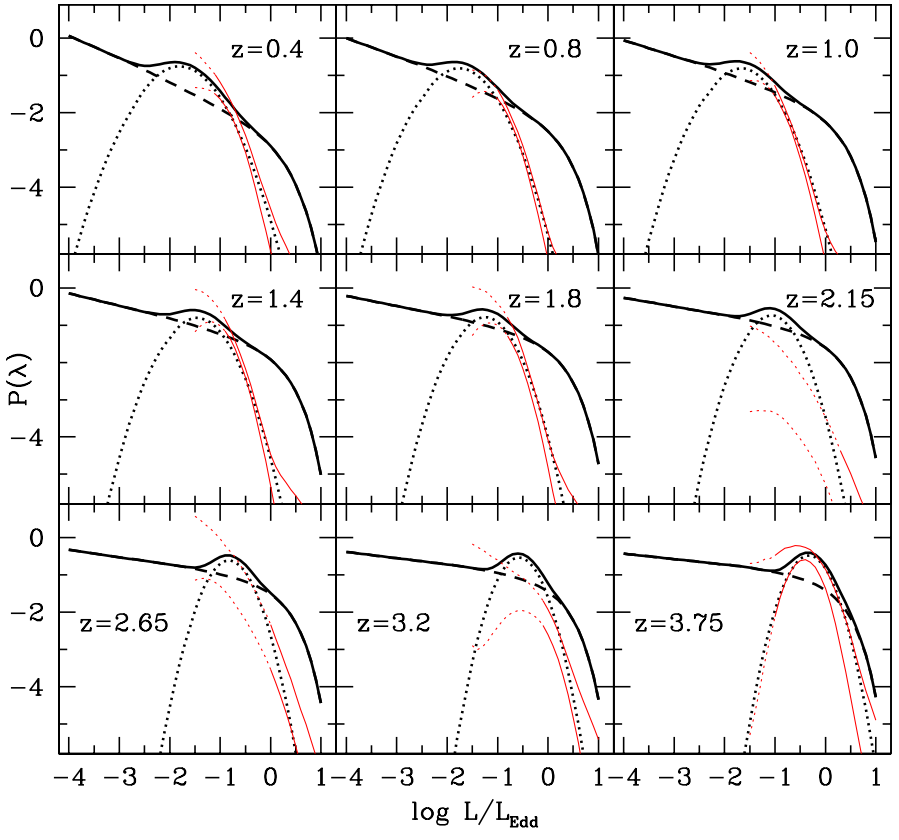

Fig. 2. Eddington ratio distribution given by Eq. (12) for SMBHs of mass $M=10^{8} M_{\odot}$ at different redshifts (black solid lines; dotted lines are for type-1 AGN and dashed lines for type-2 AGN). The Eddington ratio distributions from Kelly \& Shen (2013) with an arbritary normalization are also shown (red lines): the two lines give the uncertainty in their estimates (at $68 \%$ of probability); dotted lines denote the regions below the $10 \%$ completeness for the flux-limited SDSS sample.

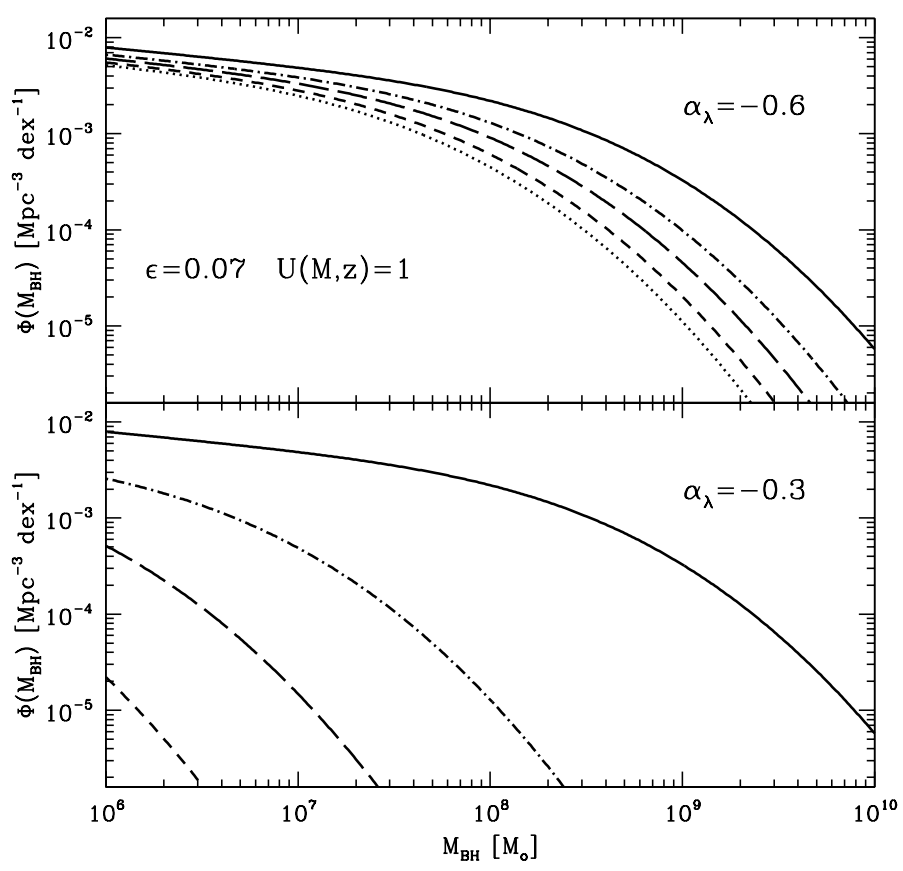

Fig. 3. SMBH mass function at redshifts $0,0.5,1,2$, and 4 (from top to bottom), assuming $U(M, z)=1$ and a power-law distribution for the Eddington ratio with a constant slope $\alpha_{\lambda}=-0.6$ (upper panel) and -0.3 (lower panel).

fraction of obscured/unobscured AGN has been largely investigated in the literature, providing evidence of an anti-correlation with nuclear luminosity (e.g., Ueda et al. 2003, 2014; Hasinger 2008; Merloni et al. 2014). Several works have also reported evidence of positive evolution of the fraction of obscured AGN 


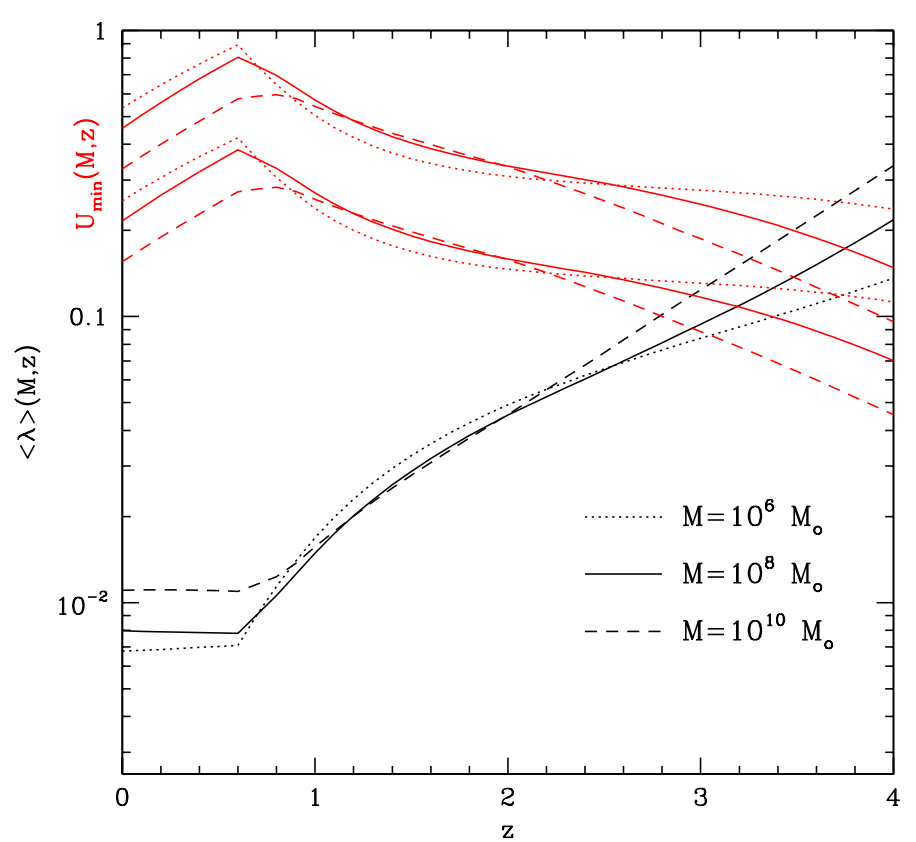

Fig. 4. Redshift evolution of the average Eddington ratio (black lines), and of the duty cycle required in order to have a growth time equal to the age of the Universe (red curves). The duty cycle is computed for $\epsilon=0.1$ (upper lines) and 0.05 (lower lines). We have considered three SMBH masses: $10^{6}$ (dotted line), $10^{8}$ (solid line), and $10^{10} M_{\odot}$ (dashed line).

with increasing redshift (La Franca et al. 2005; Hasinger 2008; Iwasawa et al. 2012; Merloni et al. 2014). In the following analysis, we adopt the parametrization provided by Ueda et al. (2014) for the fraction of obscured AGN, that is, for AGN with an intrinsic absorption $N_{\mathrm{H}} \geq 10^{22} \mathrm{~cm}^{-2}$. This is based on a combined sample of X-ray surveys of various depths, widths and energy bands. They provided the fraction of obscured AGN, $f_{\text {obs }}$, as a function of X-ray luminosity and redshift.

Because the relative abundance of type- 1 and type-2 AGN depends on the luminosity, the total Eddington ratio distribution will depend on the SMBH mass too and will take the form:

$P(\lambda \mid M, z)=a_{n}(M, z)\left[f_{\text {uno }}(L, z) P_{1}(\lambda, z)+f_{\text {obs }}(L, z) P_{2}(\lambda, z)\right]$,

where $f_{\text {uno }}(L, z)=1-f_{\text {obs }}(L, z)$ is the fraction of type-1 AGN and $f_{\mathrm{obs}}$ is in terms of the bolometric luminosity. X-ray luminosities are converted to bolometric luminosities through the bolometric corrections provided by Hopkins et al. (2007). The factor $a_{n}(M)$ is required by the normalization condition $\int \operatorname{dlog} \lambda P(\lambda \mid M, z)=1$.

Figure 2 shows $P(\lambda)$ for $M=10^{8} M_{\odot}$ at different redshifts. At low redshift, most SMBHs, including type-1 AGN, accrete at low rates, $\lambda \ll 0.1$. Increasing the redshift, the distribution for type-2 AGN becomes flatter, while for type-1 AGN this distribution becomes sharper and peaked at higher $\lambda$. At $z \gtrsim 2$, almost all the type- 1 AGN and approximately $30-50 \%$ of the total active population have $\lambda>0.01$. At this redshift, the fraction of AGN with $L \sim L_{\mathrm{Edd}}$ also becomes relevant. The dependence of the distribution on the SMBH mass is modest, and is related only to the increasing fraction of type-1 AGN with $M$.

Given the probability distribution, it is easy to compute the average Eddington ratio as a function of black hole mass and redshift (Fig. 4). We find that it is approximately $10^{-2}$ at $z<1$ and then steadily increases with redshift up to $0.1-0.3$ between

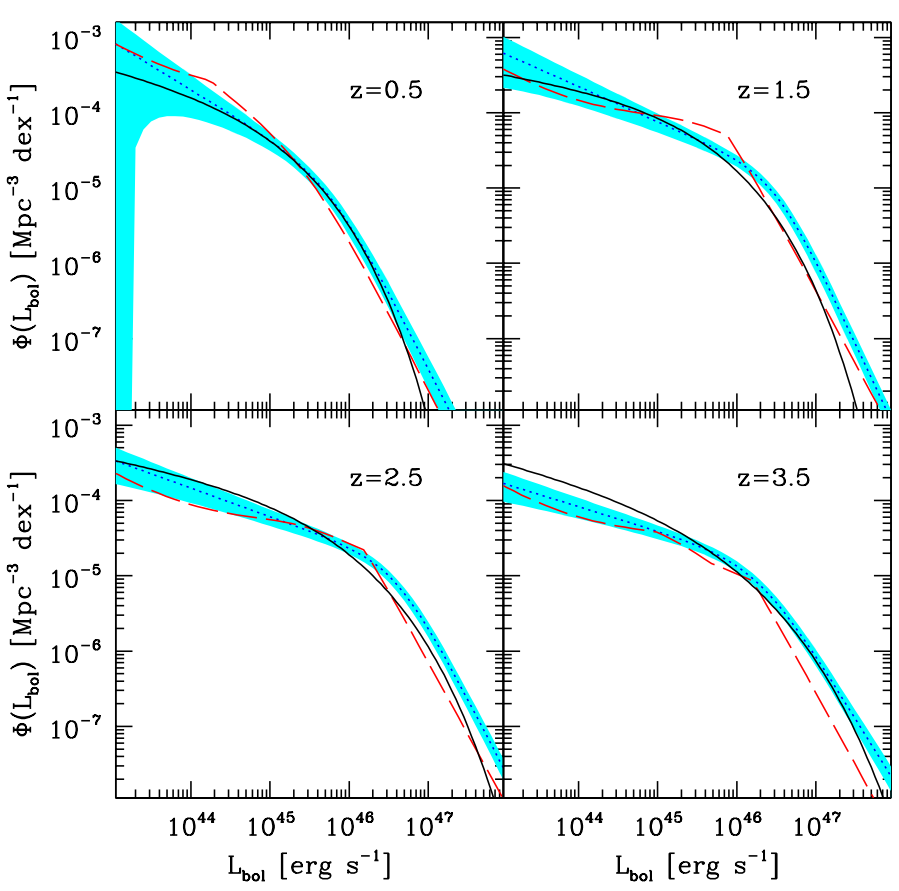

Fig. 5. Bolometric luminosity function of quasars at different redshifts from: Hopkins et al. (2007, blue dotted lines plus cyan shaded areas for the $1 \sigma$ uncertainty); Ueda et al. (2014, red dashed lines); and by Aversa et al. (2015, black solid lines).

$z=3$ and $z=4$. As expected, there is only a slight dependence on the SMBH mass.

\subsection{Quasar luminosity function}

We adopt the fitting function to the bolometric quasar luminosity function (QLF) provided by Hopkins et al. (2007). They combined a large set of measurements from the optical, soft, and hard $\mathrm{X}$-ray, and near- and mid-IR bands to determine the bolometric QLF in the redshift interval $z=0-6$. Then, they fit the observational QLF by a double power law in each redshift bin. The evolution in redshift of the best-fit parameters is also parametrized in order to provide analytical formulas to compute the QLF in a generic redshift between 0 and 6 . However, because of the increasing uncertainties in the QLF at high redshifts, we decided to restrict our analysis up to a redshift of 4 only. We use the results for the reference model, indicated as "full" in Table 3 of Hopkins et al. (2007). Based on the uncertainties of the best-fit parameters, we estimate the uncertainty on the QLF by a Monte Carlo technique (see the shaded areas in Figs. 5 and 7).

It is informative to compare the Hopkins et al. QLF, based on data available up to 2007, to QLFs derived from more recent data. In Fig. 5, we plot the bolometric luminosity functions obtained by (1) Ueda et al. (2014), based on surveys in the soft and hard X-ray bands; and (2) Aversa et al. (2015), built up from a compilation of observations in the optical and X-ray bands. We find good consistency, although the Hopkins et al. QLF is slightly higher than the other estimates at high luminosities $\left(L \gtrsim 10^{46} \mathrm{erg} \mathrm{s}^{-1}\right)$.

As an additional check, in Fig. 6, we plot the luminosity function of type-1 AGN obtained from the Hopkins et al. QLF multiplied by the fraction of unobscured AGN used in our analysis (i.e., from Ueda et al. 2014). This is compared to observational estimates of type-1 AGN luminosity functions from 


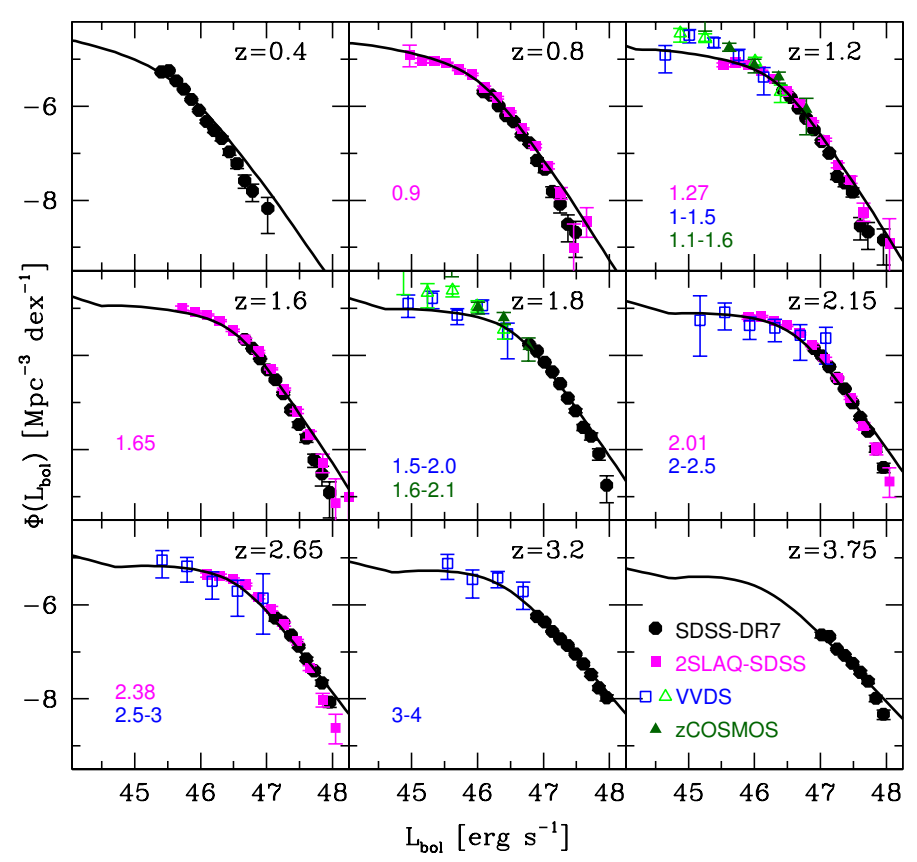

Fig. 6. Bolometric luminosity function for type-1 AGN (black lines) obtained from the QLF of Hopkins et al. (2007) rescaled by the fraction of unobscured objects of Ueda et al. (2014). The points represent measurements from different optical surveys: SDSS DR7 (black, Shen \& Kelly 2012); 2SLAQ-SDSS (magenta, Croom et al. 2009); VVDS (blue, Bongiorno et al. 2007 and green, Schulze et al. 2015); zCOSMOS (dark green, Schulze et al. 2015). The redshift for the model and for the SDSS DR7 data is marked in the upper right corner of each plot, and the redshifts for other data are marked in the lower left corner in the corresponding colors when they are different.

different optical surveys (Bongiorno et al. 2007; Croom et al. 2009; Shen \& Kelly 2012; Schulze et al. 2015) ${ }^{1}$. The agreement is, in general, extremely good. This is particularly remarkable considering the different types of data used to estimate the absorption function (X-ray band) and the type-1 AGN lumiosity function (optical band).

\subsection{Duty cycle}

As previously discussed, we define the duty cycle $U(M, z)$ as the fraction of SMBHs of mass $M$ that are active at redshift $z$, or, in other terms, as the fraction of SMBHs accreting at $\lambda \geq 10^{-4}$. Alternatively, the duty cycle of a SMBH can also be viewed as the lifetime that quasars of a given mass pass radiating at a luminosity larger than a certain value $L$ (e.g., Hopkins \& Hernquist 2009). This is a key term to solving the continuity equation and is also an important prediction of the model.

Merloni \& Heinz (2008) defined the average growth time of a SMBH as the ratio $M /\langle\dot{M}\rangle$. It measures the time it would take a SMBH of mass $M$ to double its mass if accreting at a rate $\langle\dot{M}\rangle$. In Fig. 4, we plot the value of the duty cycle needed to have a growth time equal to the age of the Universe at that redshift. This indicates the "minimum" duty cycle above which SMBHs (of mass $M$ at time $t$ ) are, on average, actively growing or, in

1 Optical magnitudes of the different data are converted before to $2500 \AA$ continuum luminosities using the relations provided by Eq. (19) in Shen \& Kelly (2012) and then to $B$-band luminosities assuming a power-law continuum slope $\alpha_{v} \simeq-0.5$. The final conversion to bolometric luminosities is done through the bolometric corrections of Hopkins et al. (2007). other terms, the BHMF is significantly evolving. We can see that phases of major growth/evolution require duty cycles close to 1 at low redshifts and, in any case, larger than 0.1 .

From an observational point of view, the duty cycle of SMBHs is difficult to estimate because it requires the simultaneous knowledge of the SMBH and AGN mass function. If we assume that all massive galaxies contain a $\mathrm{SMBH}$, then the fraction of active galaxies can be used as an observable proxy for black hole duty cycles. However, even in this way, the "measured" duty cycle will depend on the luminosity or Eddington ratio threshold of the specific observational sample.

Our approach is to assume a parametric function for the duty cycle, which is described by a double power law:

$U(M, z)=\min \left(\frac{A(z)}{\left(M / M_{0}(z)\right)^{\alpha_{l}(z)}+\left(M / M_{0}(z)\right)^{\alpha_{k}(z)}}, 1\right)$,

where we have imposed the physical condition that $U(M, z) \leq 1$. The redshift evolution of the duty cycle parameters is described by cubic polynomials,

$X(z)=a_{X}+b_{X} z+c_{X} z^{2}+d_{X} z^{3} \quad$ with $X=A, \alpha_{l}, \alpha_{k}, M_{0}$,

giving a total of 16 parameters for the duty cycle. These parameters are constrained by the condition that, at each redshift bin, the bolometric luminosity function computed by Eq. (7) has to match the observational QLF of Hopkins et al. (2007).

More in detail, given the duty cycle (i.e., chosen a set of the 16 parameters), the continuity equation is solved recursively, imposing the local SMBH mass function as an initial condition and going backwards in time from redshift 0 to 4 . At each timestep, we use the AGN MF derived from the previous timesteps to compute the update BHMF. The bolometric QLF is finally computed from Eq. (7). We employ a MCMC method to find the parameters of the duty cycle that best fit the QLF of Hopkins et al. (2007) in the redshift range $0-4$.

In principle, if the Eddington ratio distribution is fixed, the AGN MF could be directly derived from the convolution of the input $P(\lambda)$ and the QLF. The continuity equation would be used only to determine the BHMF evolution. However, this procedure does not guarantee the condition for the duty cycle to be $U(M, z) \leq 1$. We have verified, in fact, that this is not the case when the AGN MF is parametrized by a double power law (that is the most natural choice as the QLF is also well described by a double power law; see, e.g., Cao 2010; Shankar et al. 2013).

As a consistency test, we developed another method to determine the duty cycle parameters of Eq. (13) that does not rely on any functional form for the redshift dependence of the parameters. In this case, the duty cycle is assumed to be constant in small redshift intervals of $\Delta z=0.1$. In each redshift bin, $z_{i}$, the four free parameters of $U(M, t)$ (i.e., $A, \alpha_{l}, \alpha_{k}$ and $\left.M_{0}\right)$ are determined by finding the best fit to the observational QLF at $z=z_{i-1}+\Delta z / 2$. In general, the two methods provide consistent results, although the former is able to provide better fits to the QLF at high redshifts, especially when the BHMF is strongly evolving with time (i.e., for low values of the radiative efficiency).

\section{Results}

In this section, we present the results of the model in terms of BHMF, duty cycle, and AGN MF (Fig. 9). The average radiative efficiency of SMBHs is a free parameter and we solved the continuity equation for different values of $\epsilon$. In addition, we considered two different inputs of the local mass function, obtained 

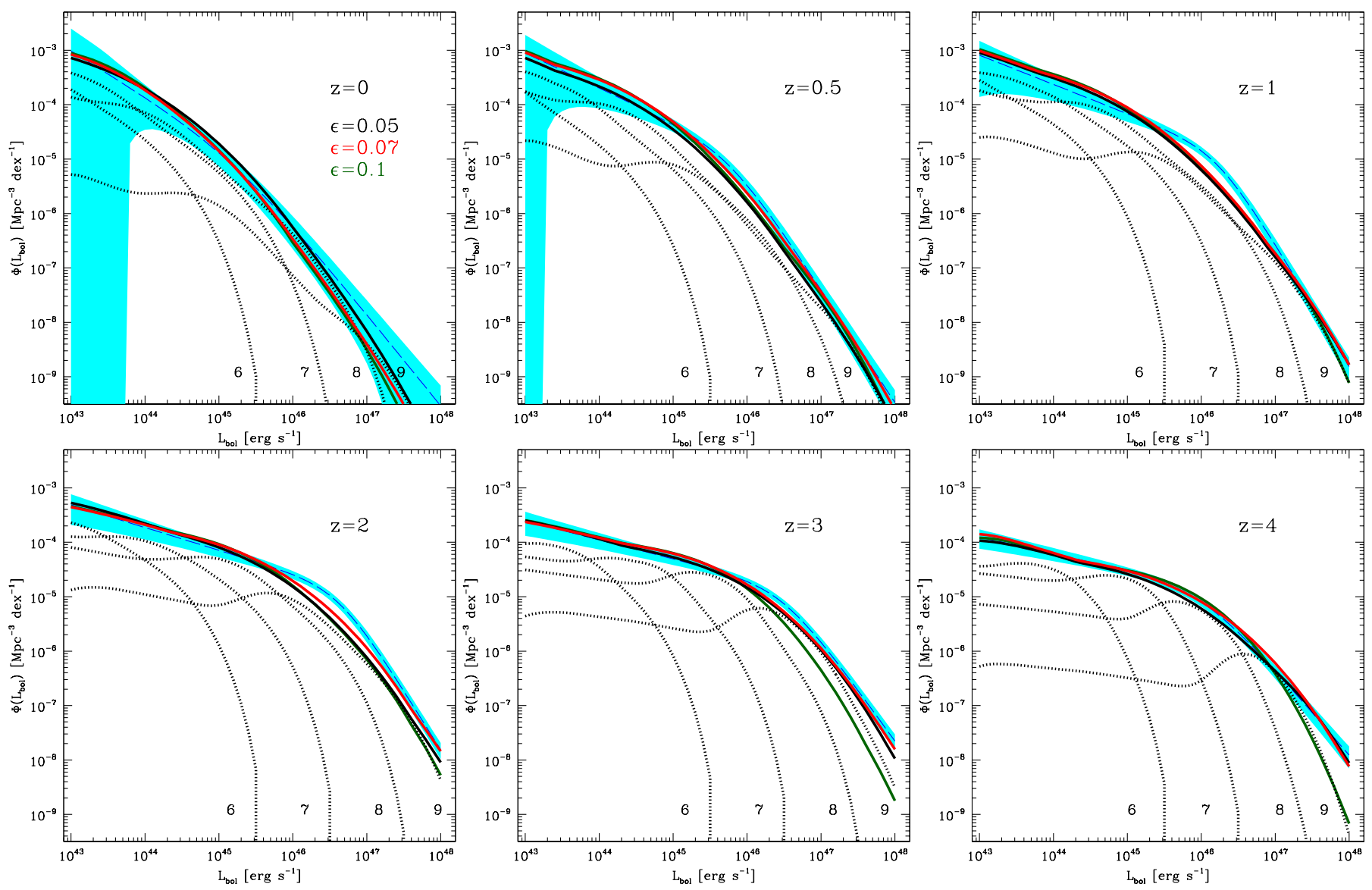

Fig. 7. Bolometric quasar luminosity function at different redshifts predicted by the models (solid black, red, and green lines for $\epsilon=0.05,0.07$, and 0.1 , respectively), and compared with the estimates from Hopkins et al. (2007, blue dashed lines plus cyan shaded areas for the $1 \sigma$ uncertainty). Black dotted lines show the contribution to the LF from SMBHs with mass in the interval indicated in the plots (e.g., "6" corresponds to a mass interval $\left[5 \times 10^{5}, 5 \times 10^{6}\right) M_{\odot}$ ) for the model with $\epsilon=0.05$.

by convolving the Schechter function in Eq. (8) with a Gaussian scatter of 0.3 (LMF03) and $0.5 \mathrm{dex}$ (LMF05).

Three models are described in detail: the LMF05 local BHMF and $\epsilon=0.05$ and 0.07; the LMF03 local BHMF and $\epsilon=0.1$. They provide the best fit to the QLF and show more "reliable" behaviors in term of black hole MF evolution with respect to other model inputs (see Appendix A). Below, we refer to these models just for the different value of the radiative efficiency, but encourage the reader to also keep in mind the different local BHMF used. In Appendix A, we show results with different combinations of $\epsilon$ and the local BHMF, and we provide a general discussion about the uncertainties in the model predictions.

\subsection{Bolometric quasar luminosity function}

In Fig. 7, we compare the QLF estimated by Hopkins et al. (2007) with results from the three models. In general, the models accurately reproduce the observational LF, although the transition between the low- and high-luminosity tails is smoother in the models at $1 \lessgtr z \lesssim 2$. At redshifts $z>2$, the case with $\epsilon=0.1$ tends to underestimate the observational QLF for $L \gtrsim 10^{47} \mathrm{erg} \mathrm{s}^{-1}$, due to the very low number density of SMBHs with $M>10^{9} M_{\odot}$ predicted by the model (see below).

In Fig. 7, we also plot the contribution to the QLF from SMBHs in different ranges of mass. The shape of these contributions reflects the Eddington ratio distribution. For a small interval of masses, in fact, the QLF is simply $\Phi(L) \approx$ $P(\lambda \mid M) \Phi_{\mathrm{AGN}}(M)$. The peak of the log-normal distribution of type-1 AGNs is clearly visible and, at high redshifts, we can associate a narrow range of masses to each luminosity for the SMBHs that mainly contribute to the QLF.

Finally, we derive the time evolution of the black hole accretion rate density (BHAD), $\Upsilon_{\mathrm{BHAD}}$, as predicted by the models. This quantity is defined as

$\Upsilon_{\mathrm{BHAD}}(z)=\int_{0}^{\infty} \frac{1-\epsilon}{\epsilon c^{2}} L \Phi(L, z) \mathrm{d} \log L$.

In Fig. 8, we plot $\Upsilon_{\text {BHAD }}$, normalized by the factor $\epsilon /(1-\epsilon)$ in order to remove the dependence on the radiative efficiency. For comparison, we plot the BHAD obtained by integrating the observational QLFs of Hopkins et al. (2007), Ueda et al. (2014), Aversa et al. (2015). We also report the estimates of Delvecchio et al. (2014), using a sample of far-infrared galaxies from Herschel. Our results are highly consistent with these estimates. At redshift $z \sim 2$, where the peak of the accretion rate density is found, our models underestimate the BHAD of Hopkins et al. (2007) by $20-40 \%$, but are highly compatible with estimates of Delvecchio et al. (2014), Aversa et al. (2015).

\subsection{Mass function and duty cycle of $\mathrm{SMBHs}$}

In Fig. 9, we show the model predictions for the evolution of the BHMF between redshift 0 and 4 . Our results agree with 


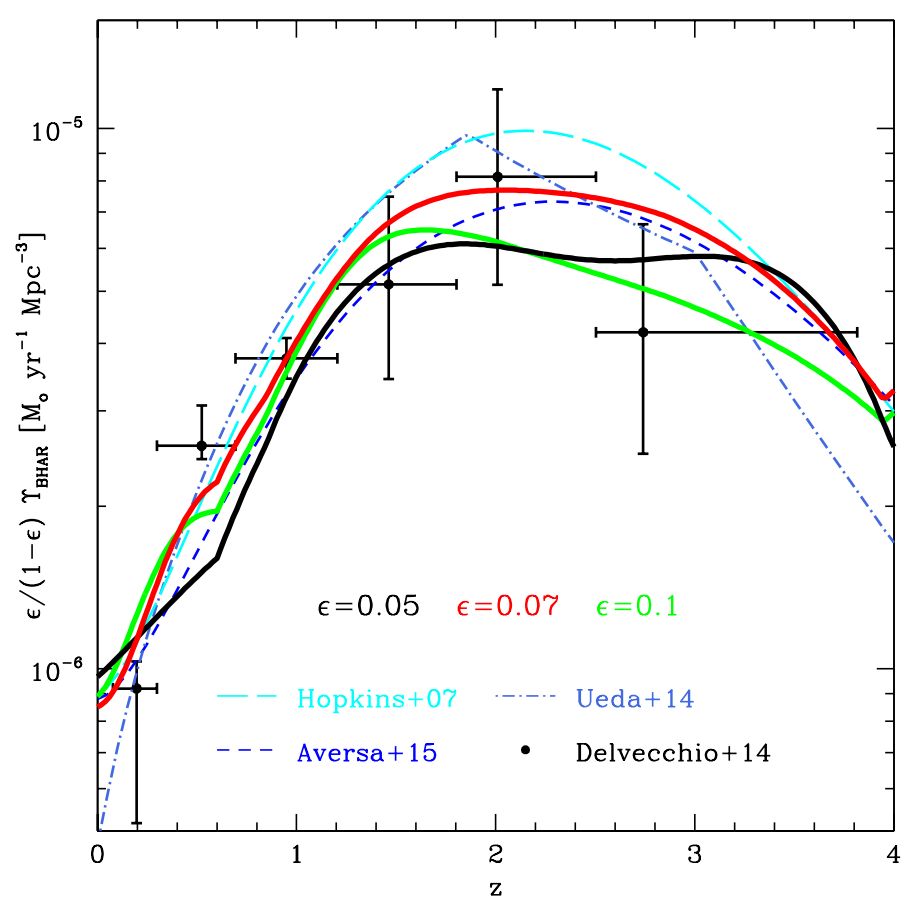

Fig. 8. Black hole accretion rate density (multiplied by $\epsilon /(1-\epsilon)$ ) from our reference models with $\epsilon=0.05,0.07$, and 0.1 (solid thick lines); these are compared with the BHAD obtained from different bolometric LFs (as indicated in the plot).

an anti-hierarchical growth of SMBHs ("cosmic downsizing"), where most of the low-mass SMBHs were formed later in time than high-mass SMBHs (e.g., Granato et al. 2001; Ueda et al. 2003; Marconi et al. 2004; Merloni \& Heinz 2008; Shankar et al. 2009). More quantitatively, at low redshifts, the mass function evolves only at low/intermediate masses, and it decreases by approximately $30-60 \%$ from $z=0$ to 1 for objects of $10^{6}-10^{7} M_{\odot}$. On the contrary, the number density of very massive SMBHs $\left(M \gtrsim 10^{9} M_{\odot}\right)$ remains practically unchanged up to $z=1$, and then undergoes a significant evolution.

The details of the SMBH cosmic history depend strongly on the radiative efficiency. If we compare the results for $\epsilon=0.05$ and 0.07 , we note a much faster evolution after redshift 2 if $\epsilon=0.05$. In this case, the BHMF drops by one order of magnitude between $z=2$ and 4 in the whole range of masses. Instead, both the models with $\epsilon=0.1$ and 0.07 predict a similar moderate evolution of the BHMF: low-mass objects evolve mainly at redshifts $0-2$, while SHBHs with $M>10^{8} M_{\odot}$ evolve between redshifts 1 and 3 . In both cases, the MF does not significantly change between $z=3$ and 4 , and at these redshifts, it is only a factor $\lesssim 10$ lower than the local BHMF. The main difference between the two models is the larger number density of very massive SMBHs predicted by the model with $\epsilon=0.07$; an effect of the different local BHMF employed.

The duty cycle is also shown in Fig. 9 and the best-fit parameters of Eqs. (13), (14) are provided in Table 1. We can point out a general trend in all three cases: at $z \lesssim 1$ the duty cycle is $\sim 1$ at $M \lesssim 10^{7} M_{\odot}$ and then decreases with the SMBH mass; at $z>1$, the duty cycle is less dependent on $M$, and for $\epsilon=0.07$ and 0.1 (we note the similar behavior of $U(M, z)$ ), it is almost constant at $z \gtrsim 2$ and decreasing with time. On the contrary, for $\epsilon=0.05$, the duty cycle always increases with redshift and most SMBHs are active at $z=4$. In this case, because of the strong evolution of the BHMF, a high fraction of active SMBHs is required in order to fit the QLF at high redshifts.
Table 1. Best-fit parameters of the duty cycle (see Eqs. (13), (14)) for the different values of the radiative efficiency.

\begin{tabular}{cccccc}
\hline \hline$\epsilon$ & $X$ & $a_{X}$ & $b_{X}$ & $c_{X}$ & $d_{X}$ \\
\hline 0.1 & $\mathrm{~A}$ & 0.956 & -1.337 & 0.383 & -0.0450 \\
& $\alpha_{l}$ & 0.250 & -0.211 & 0.0434 & $-1.82 \mathrm{e}-04$ \\
& $\alpha_{k}$ & 1.159 & -1.571 & 1.354 & -0.0256 \\
& $M_{0}$ & 4.884 & 4.648 & -1.284 & 0.109 \\
\hline 0.07 & $\mathrm{~A}$ & 0.634 & -0.803 & 0.151 & -0.0122 \\
& $\alpha_{l}$ & 0.944 & -0.706 & 0.194 & 0.438 \\
& $\alpha_{k}$ & 0.0456 & 0.149 & -0.132 & 0.0259 \\
& $M_{0}$ & 4.965 & 4.913 & -1.545 & 0.183 \\
\hline 0.05 & $\mathrm{~A}$ & -0.0104 & 0.309 & -0.0659 & $-3.70 \mathrm{e}-03$ \\
& $\alpha_{l}$ & 1.012 & -0.335 & 0.0123 & $2.06 \mathrm{e}-03$ \\
& $\alpha_{k}$ & 0.195 & -0.0570 & 0.0348 & $2.10 \mathrm{e}-03$ \\
& $M_{0}$ & 7.389 & 0.714 & -0.0373 & $-8.98 \mathrm{e}-03$ \\
\hline
\end{tabular}

Given the BHMF and the duty cycle, computing the mass function for active SMBHs (i.e., the AGN MF) is straightforward. All the models predict very similar AGN MFs (see Figs. 9 and 10, where the type-1 and type- 2 AGN MFs are given separately). The only exception is at $M \gtrsim 10^{9} M_{\odot}$, where the model with $\epsilon=0.1$ predicts a significantly lower number density of active SMBHs. However, because it also tends to underestimate the observational QLF at high luminosities, we expect that the actual MF is larger than predicted by the model in this range of masses. This issue may be related to the use of the LMF03 local BHMF.

From Fig. 9 we can note that the number density of low mass AGN decreases by more than an order of magnitude between $z=$ 0 and 4. On the contrary, for massive $\operatorname{AGN}\left(M>10^{8} M_{\odot}\right)$, the number density peaks at redshifts $1-2$ and then rapidly decreases (by a factor $\sim 10$ ) up to $z=4$. This is a signature of the cosmic downsizing of AGN. From Fig. 10, we also note the increase of the fraction of type-1 AGN with the SMBH mass.

It is important to stress that, contrary to what occurs for the BHMF, the AGN MF is almost insensitive to the choice of the local mass function and of the value of $\epsilon$. This result can be understood considering that our method, and standard continuity equation approaches, in general, use the QLF as observational constraints, that depends, through Eq. (7), on the AGN MF, but not on the BHMF. If the Eddington ratio distribution for SMBHs is known, the QLF puts strong constraints on the AGN mass function, at least for masses $M \lesssim 10^{9} M_{\odot}$ (more massive SMBHs give a small contribution to the QLF, as shown in Fig. 7). On the other hand, the continuity equation method is not able to constrain details of the SMBH growth history that depend on the model parameters (see also Caplar et al. 2015; Veale et al. 2014). Only a direct measurement of the BHMF at redshifts $z \gtrsim 2$ could break these degeneracies, giving important constraints on the average radiative efficiency, for example.

A quantity that is useful for gaining information on the typical accretion rate of SMBHs is the growth time, that is, the ratio $M /\langle\dot{M}\rangle$ (Merloni \& Heinz 2008). Figure 11 shows the redshift evolution of the growth time as a function of SMBH mass. In each panel, we draw the age of the Universe at that time as a reference. SMBHs with growth time longer than the age of the Universe are not experiencing a major growth phase, which must have necessarily happened in the past. On the contrary, objects with growth times shorter than that are actively growing. Looking at Fig. 11, we see that only small mass SMBHs $\left(M \lesssim 10^{7} M_{\odot}\right)$ can be actually growing in mass at low redshifts 

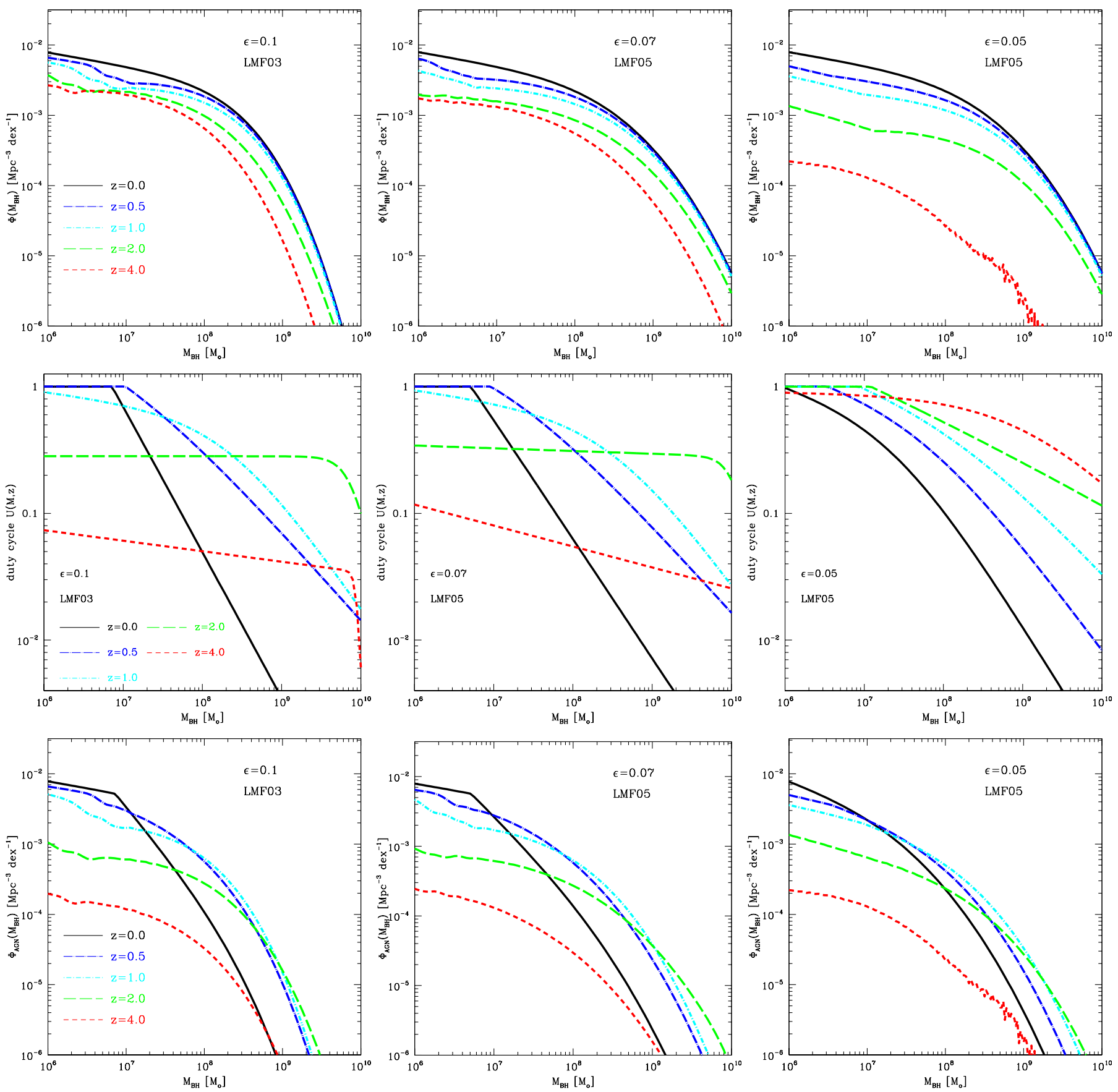

Fig. 9. Top panels: SMBH mass function between redshift 0 and 4 as predicted by the models with different choices of the radiative efficiency and the local mass function. At $z<1$, the BHMF evolves only at $M<10^{8} M_{\odot}$, while number density of very massive SMBHs undergoes a strong evolution a higher redshifts. At $z>1$, details of the SMBH cosmic history strongly depend on the radiative efficiency. Central panels: duty cycle $U(M, z)$ as predicted by the models. At $z<1$, the duty cycle is $\sim 1$ for SMBHs of $M<10^{7} M_{\odot}$ and then decreases with the mass. The behavior of the duty cycle is very similar for the models with $\epsilon=0.1$ and 0.07 , increasing from redshift 0 to 1 , and decreasing at $z>2$. For $\epsilon=0.05$, it always increases and most SMBHs are active at $z \sim 4$. Bottom panels: AGN MF as predicted by the models. This is almost insensitive to the choice of the local mass function and to the value of the radiative efficiency. The number density of active SMBHs with $M \geq 10^{8} M \odot$ peaks at redshifts $1-2$ and then rapidly decreases.

(see also Fig. 4). This is expected according to the downsizing behavior of SMBHs, in which only low-mass SMBHs are still accreting mass and growing in the local Universe or at low redshift; in agreement with observational constraints (Heckman et al. 2004; Greene \& Ho 2007; Goulding et al. 2010; Schulze $\&$ Wisotzki 2010). The main epoch of growth for SMBHs is around redshift $1.5-3$. At these redshifts, the growth time is almost independent of $M$ and below the age of the Universe. At $z>3$, the growth time becomes typically larger than the age of the Universe, except for $\epsilon=0.05$. In this case, the growth time steadily decreases and SMBHs are active and growing independently of their mass, up to at least redshift 4.

Figure 12 illustrates the evolution of the integrated SMBH mass density, $\rho_{\mathrm{BH}}=\int \log (M) M \Phi_{\mathrm{BH}}(M)$, as predicted by the models. The interesting feature is the very different time evolution of $\rho_{\mathrm{BH}}$ between the model with $\epsilon=0.05$ and $0.07 / 0.1$. In the latter, the evolution is modest, decreasing by a factor 


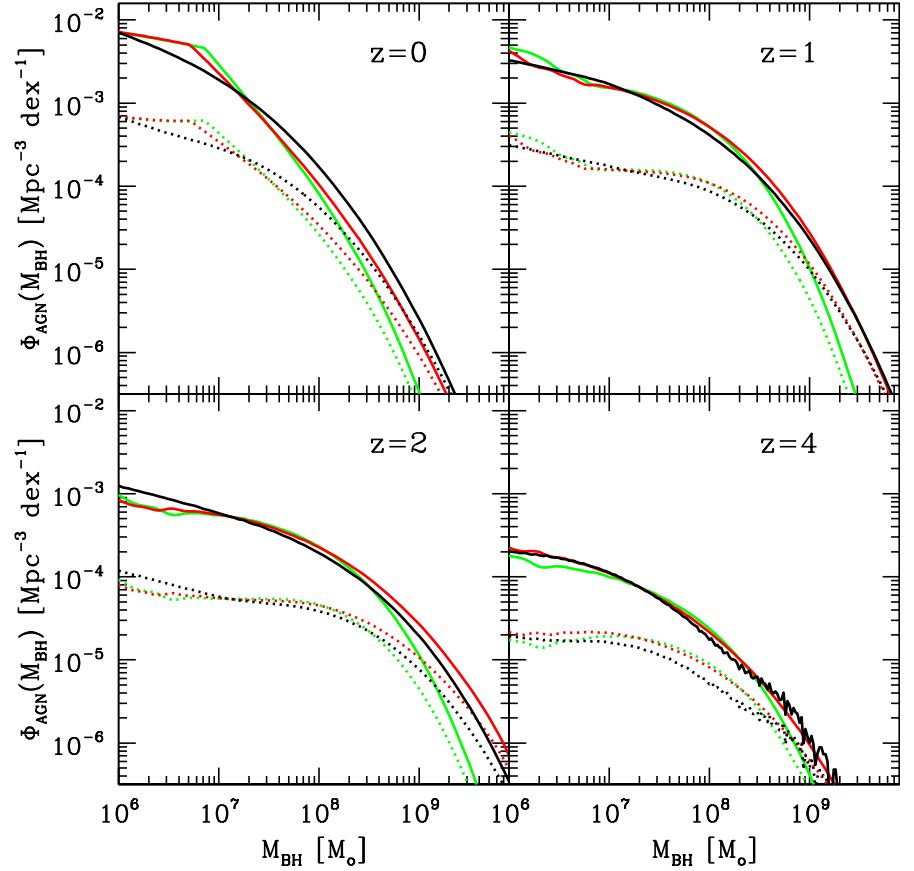

Fig. 10. Mass function for type-1 (dotted lines) and type-2 (solid lines) AGN according to the models with $\epsilon=0.1$ (green lines), $\epsilon=0.07$ (red lines), 0.05 (black lines). The AGN mass functions are practically independent of the model in the all redshift range. The fraction of type-1 AGN increases with mass.

approximately 5 from redshift 0 to 4 . On the contrary, for $\epsilon=$ 0.05 , the mass density is lower than $10^{4} M_{\odot} \mathrm{Mpc}^{-3}$ at $z=4$, that is, approximately two orders of magnitude below the local density. This behavior is in very good agreement with observational findings from Ueda et al. (2014) obtained from a compilation of AGN X-ray luminosity surveys assuming a radiative efficiency of 0.05 .

The different evolution of the BHMF, as predicted by our models, has important implications on models for the seed population of SMBHs (see, e.g., Volonteri et al. 2008; Volonteri 2010). Our results with large radiative efficiencies $(\epsilon \geq 0.07)$ indicate that a large fraction of SMBHs were already formed at $z>4$. This implies very massive primordial black hole seeds, as expected from the direct collapse of supermassive stars (e.g., Koushiappas et al. 2004; Begelman et al. 2006; Lodato $\&$ Natarajan 2007; Devecchi \& Volonteri 2009). The case with $\epsilon=0.05$ is instead more compatible with models in which primordial black holes originate from stellar mass progenitors (remnants of the first, Population III, stars; see e.g., Abel et al. 2000; Bromm et al. 2002) and that predict negligible SMBH mass density at high redshifs.

If we consider active SMBHs, Fig. 12 shows a peak in the mass density of type- 2 and type-1 AGN at redshifts of approximately $0.5-1.5$. The peak is more pronounced in type-1 AGN due to the increase of their fraction with luminosity and redshift. As expected, the evolution of the AGN mass density is almost independent of the model. The mass density is somewhat lower for $\epsilon=0.1$, an effect of the sharper drop of the mass function at $M \gtrsim 10^{9} M_{\odot}$, observed in Fig. 10 .

Observational estimates of the quasar mass function and of the ERDF are a promising test for model predictions. Nevertheless, direct comparison between model and observations is not trivial due to selection effects in quasar samples. A standard approach to compute the MF and ERDF is based on

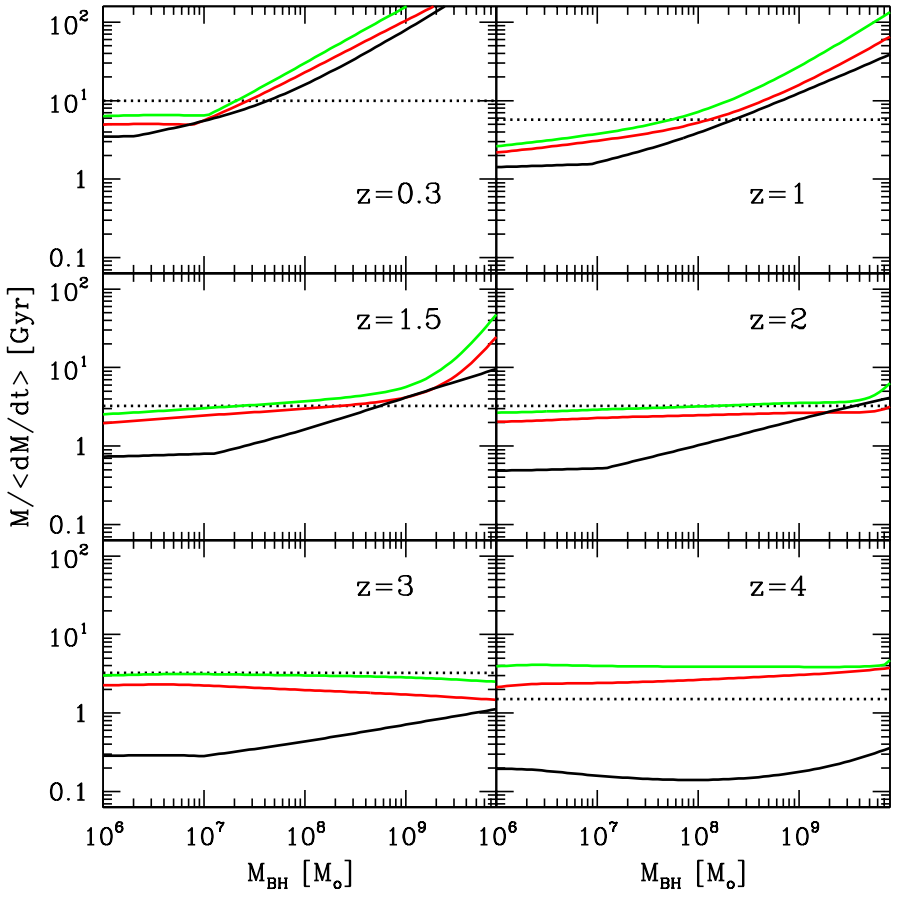

Fig. 11. Redshift evolution of the growth time as a function of SMBH mass. In each panel, the horizontal dotted line marks the age of the Universe at that redshift. Lines indicate the different values of $\epsilon$ : black, red and green lines are for $\epsilon=0.05,0.07$ and 0.1 , respectively. The main epoch of growth is at $1<z<3$. At later time, only low-mass SMBHs can be actually growing in mass, while at earlier time only in the model with $\epsilon=0.05$ the growth time is much lower than the age of the Universe.

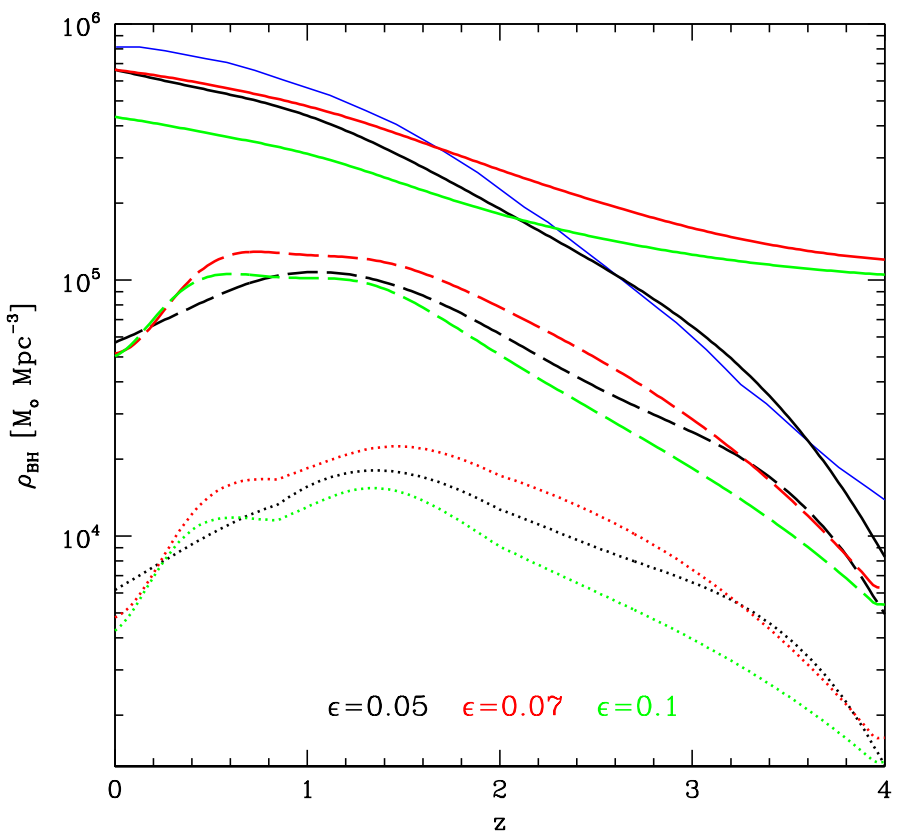

Fig. 12. Mass density of SMBHs (solid lines), active SMBHs (dashed lines), and type-1 AGN (dotted lines) for the three values of the radiative efficiency indicated in the plot. The thin blue solid line corresponds to results from Ueda et al. (2014). If $\epsilon=0.1$ or 0.07 , the evolution of the mass density is relatively modest. On the contrary, if $\epsilon=0.05$, the mass density at $z=4$ is two orders of magnitude lower than the local one. The AGN mass density has similar evolution for the three cases, with a peak at approximately $z=0.5-1.5$. 

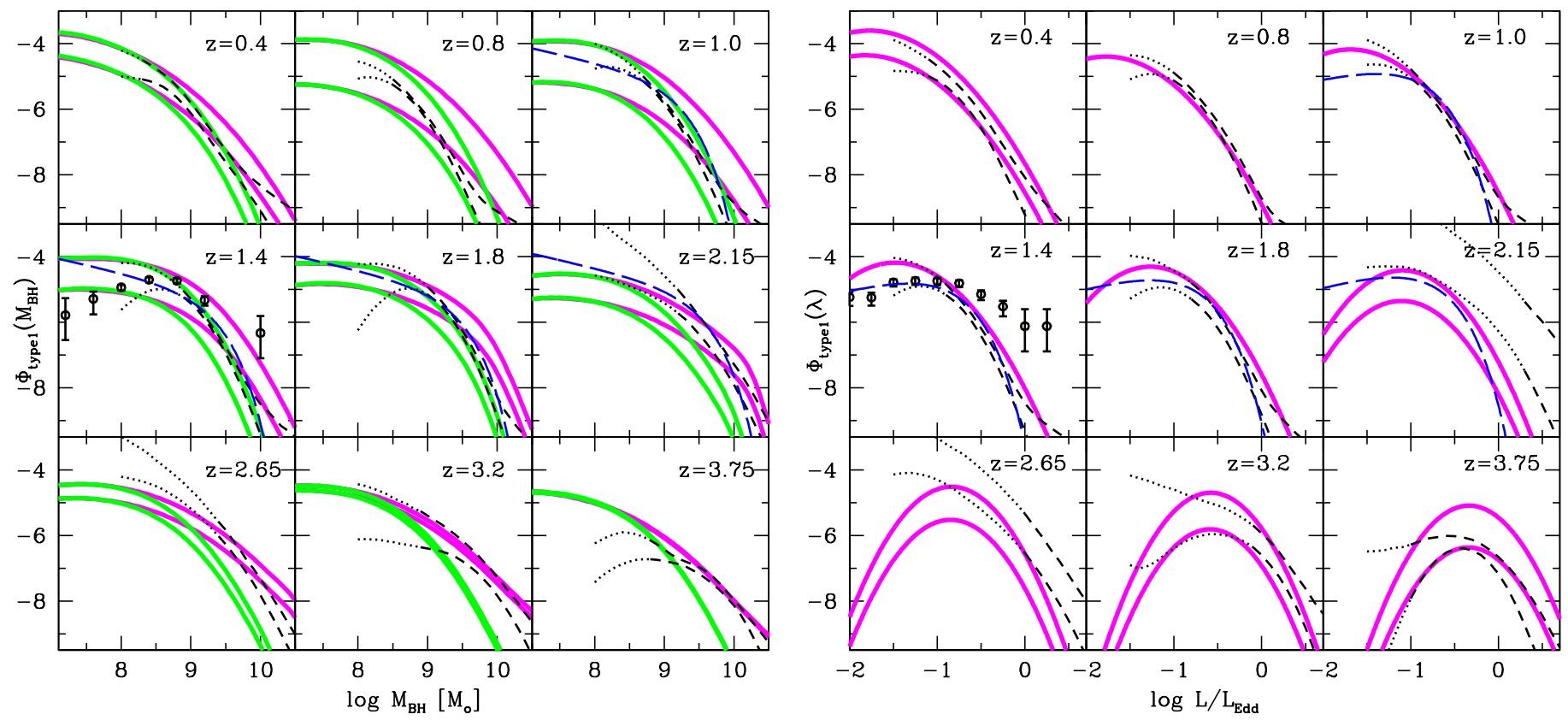

Fig. 13. Left panel: MFs for type-1 AGN computed from the model and compared with observational estimates. Solid thick lines are for the model with $\epsilon=0.07$ (magenta lines) and $\epsilon=0.1$ (green lines), assuming the values of $\lambda_{\min }$ reported in Table 2. Observational constraints are from Schulze et al. (2015, at $1 \leq z \leq 2.15$; blue long dashed lines), Nobuta et al. (2012, at $z=1.4$; black open points) and Kelly \& Shen (2013, black dashed/dotted lines; the two lines define the region of $68 \%$ probability, and the dotted lines denote that the completeness for the SDSS sample is below 10\%). Right panel: ERDF for type-1 AGN, as in the left panel. Here we plot only the model with $\epsilon=0.07$.

the $1 / V_{\max }$ estimator with the same volume weights as for the QLF (e.g., Wang et al. 2006; Vestergaard et al. 2008; Vestergaard \& Osmer 2009; Schulze \& Wisotzki 2010; Nobuta et al. 2012). This method has shown to suffer from severe incompleteness due to active SMBHs below the flux limit of the survey that are not taken into account (see discussion in Kelly et al. 2009; Schulze \& Wisotzki 2010; Schulze et al. 2015). Uncertainties and scatter in the relation to estimate SMBH masses have also to be considered for a proper MF determination. Several studies have estimated the MF and ERDF for type-1 AGN, employing statistical methods to properly account for the survey selection function and the uncertainties in SMBH mass estimates (Kelly et al. 2009; Schulze \& Wisotzki 2010; Shen \& Kelly 2012; Nobuta et al. 2012; Kelly \& Shen 2013; Schulze et al. 2015). Their results are model dependent because they use analytic functions to describe the bivariate distribution function of $M$ and $\lambda$.

Kelly \& Shen (2013) determined the MF and the ERDF for a sample of type-1 AGN from the SDSS at redshifts $0.4<z<5$. They found that the sample becomes significantly incomplete ( $10 \%)$ at $M \lesssim 3 \times 10^{8} M_{\odot}$ or $\lambda \lesssim 0.07$, with some variation with redshift. In Fig. 13, we plot their results and compare them with predictions of our models. At a fixed redshift, we compute the MF of type-1 AGN as:

$$
\Phi_{\mathrm{AGN}}^{\mathrm{type} 1}(M)=\Phi_{\mathrm{AGN}}(M) a_{n}(M) \int_{\log \lambda_{\min }}^{1} \operatorname{dlog} \lambda f_{\mathrm{uno}}(L) P_{1}(\lambda),
$$

and the ERDF as:

$$
\Phi_{\mathrm{AGN}}^{\mathrm{type} 1}(\lambda)=P_{1}(\lambda) \int_{\log M_{\mathrm{min}}}^{11} \operatorname{dlog} M a_{n}(M) f_{\mathrm{uno}}(L) \Phi_{\mathrm{AGN}}(M),
$$

where $f_{\text {uno }}(L)$ is the fraction of type-1 AGN with luminosity $L$, and $M_{\min }\left(\lambda_{\min }\right)$ is the minimum SMBH mass (Eddington ratio) associated to the flux-limited survey used in Kelly \& Shen (2013). The actual values of $M_{\min }$ and $\lambda_{\min }$ are not well determined and we choose two values that should represent upper and lower limits for them (see Table 2).
Table 2. Values of $M_{\min }$ and $\lambda_{\min }$ used in Eqs. (16) and (17) to compute AGN MF and ERDF for the comparison with Kelly \& Shen (2013) estimates.

\begin{tabular}{ccc}
\hline \hline Redshift & $\log M_{\min }\left[M_{\odot}\right]$ & $\lambda_{\min }$ \\
\hline 0.4 & $7 ., 8$. & $0.01,0.05$ \\
$(0.4,2)$ & 8. & $0.01,0.1$ \\
$>2$ & $8 ., 9$. & $0.07,0.2$ \\
\hline
\end{tabular}

\subsection{Comparison with observations}

Figure 13 shows our predictions for the models with $\epsilon=0.1$ and 0.07 (the results are similar for $\epsilon=0.05$ and 0.07 models). At redshifts $z<2$, the MF is relatively highly dependent on the choice of $\lambda_{\min }$ due to the broad shape of the ERDF that peaks at $0.01 \leq \lambda<0.1$. The comparison with observations is more interesting at high redshifts where the choice of $\lambda_{\min }$ is less important. In general, the models are reasonably consistent with observational estimates of Kelly \& Shen (2013). The case with $\epsilon=0.1$ better reproduces the shape of the observational MF at low redshifts, but fails at high redshifts where it strongly underestimates the observations for $M \gtrsim 10^{9} M_{\odot}$. Viceversa, models with $\epsilon=0.05 / 0.07$ fit the data at $z \gtrsim 2$ relatively well, but at low redshifts, the predicted MF seems to decrease too slowly with the mass and gives an excess of high-mass type-1 AGN. We must note, however, that SMBHs with $M \gtrsim 5 \times 10^{9} M_{\odot}$ give a negligible contribution to the QLF at $z<2$ (see Fig. 7), and the AGN MF is therefore poorly constrained by the analysis at these masses. This argument does not work for the discrepancy observed in the $\epsilon=0.1$ model: the fast decline of the MF at high redshifts cannot be reconciled with the observations even assuming duty cycles equal 1 .

Concerning the ERDF, we reiterate that the model uses a log-normal function that fits the shape of the Eddington ratio distribution determined by Kelly \& Shen (2013) at the different 


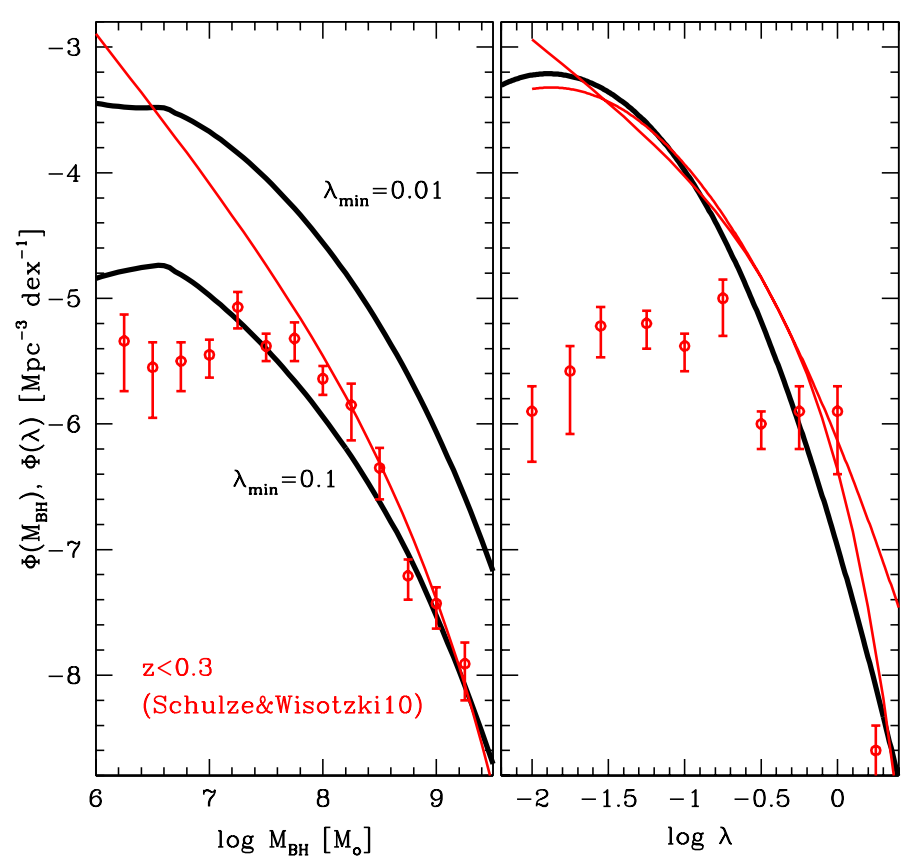

Fig. 14. Local $(z=0.1)$ BHMF (left panel) and ERDF (right panel) for type-1 AGN predicted by the model with $\epsilon=0.07$ (solid black thick lines). The BHMF is computed taking $\lambda_{\min }=0.01$ and 0.1 . The ERDF is computed for $M_{\min }=10^{6} M_{\odot}$. Red points are the distribution functions derived by Schulze \& Wisotzki (2010) directly from data, while thin red lines are their best fits, taking the sample selection function into account.

redshifts. Therefore, in Fig. 13, we simply verify whether or not the amplitude of the ERDF computed by Eq. (17) is consistent with observational results. The agreement is good with $M_{\text {min }}=10^{8} M_{\odot}$, apart from the first and last redshift bin in which $M_{\min }=10^{7}$ and $10^{9} M_{\odot}$, respectively, provide a better fit to the data. The only discrepancies we observe are at $z=2.15$ and 2.65, where our predictions are significantly lower than the data. However, Kelly \& Shen (2013) questioned the reliability of their estimates at these redshift bins because they found an apparent discontinuity across $z \sim 2$ in the number densities of type- 1 AGN radiating at $\lambda \gtrsim 0.05$. They attributed the discontinuity to systematic errors in the incompleteness correction. This, however, could also be due to the different mass estimator used before and after $z \sim 2$ (Schulze et al. 2015).

Our predictions on the ERDF at $z \sim 2$ are instead consistent with results from Schulze et al. (2015, blue lines in Fig. 13). They combined large area SDSS data with two deep, small-area surveys (VVDS and zCOSMOS) to cover a wide range of luminosities at redshifts $1 \lesssim z \lesssim 2$. They use a maximum likelihood approach to fit a parametric bivariate distribution function in the intervals of $-2<\log \lambda<1$ and $7<\log \left(M / M_{\odot}<11\right.$. Their estimates agree well with the Kelly \& Shen (2013) MF and ERDF at redshift $z<2$. In Fig. 13, we also consider the results from Nobuta et al. (2012), that used the Subaru XMM-Newton Deep Survey (SXDS) to determine the MF and ERDF at $z \sim 1.4$. Their sample is X-ray selected and extends significantly deeper than SDSS, over an area of $\sim 1.0 \mathrm{deg}^{2}$. Their MF is in agreement with the other estimates in the mass range $8-9.5 M_{\odot}$, while they show a turnover at lower masses not confirmed by the other data. Some discrepancies are also observed in the ERDF that gives a much larger probability for high accretion SMBHs.

Finally, in Fig. 14, we consider the local MF and ERDF for type-1 AGN computed by Schulze \& Wisotzki (2010). They used a sample of local $(z<0.3)$ broad line AGN from the Hamburg/ESO Survey. In Fig. 14, we report the MF and ERDF directly determined from the data (red points) and after the incompleteness correction through a maximum likelihood approach (red lines). The model seems to be in better agreement with the observational MF before the incompleteness correction (it requires $\lambda_{\text {min }} \simeq 0.1$ ). On the contrary, the predicted ERDF is only consistent with data after the incompleteness correction.

\section{Conclusions}

In this paper, we study the time evolution of the mass function of SMBHs and of their active population (i.e., SMBHs with $\lambda \geq 10^{-4}$ ) by the continuity equation, backwards in time from the present to redshift 4 . In our approach, we distinguish active SMBHs between type-1 and type- 2 AGN. The Eddington ratio distribution of the two classes is chosen on the basis of recent observational estimates, assuming a log-normal distribution and a truncated power law for type-1 and type- 2 AGN, respectively. The duty cycle of SMBHs, as a function of redshift and mass, is instead determined from the best fit of the observational quasar luminosity functions of Hopkins et al. (2007).

We also investigate the dependence of the SMBH and AGN evolution on the main assumptions/inputs employed in the analysis. These are: (1) the value of the average radiative efficiency of SMBHs, which is the only free parameter of the model; (2) the local SMBH mass function; and (3) the Eddington ratio distribution for type-1 and type-2 AGN. Below we summarize and discuss our results.

- The evolution of the BHMF, especially at $z \gtrsim 2$, is very sensitive to the value of the average radiative efficiency. This is clearly shown by comparing the results using $\epsilon=0.07$ and 0.05: with the lower radiative efficiency, the evolution of the BHMF is significantly stronger at $z \gtrsim 2$, and at $z=4$ the mass function is one order of magnitude lower than that with $\epsilon=0.07$. For larger radiative efficiencies, we find very little evolution in the MF, especially at low masses, and the number density of SMBHs (of $M \lesssim 10^{9} M_{\odot}$ ) increased by only a factor of approximately 4 (2) from $z=4$ to 0 if $\epsilon=0.1$ (0.15).

- Radiative efficiencies much larger than 0.1 seem to be discarded by our model. In this case, in fact, the BHMF is almost constant over time, implying that most SMBHs we observe at low redshifts were already formed before redshift 4 . For large radiative efficiences, a significant evolution in time of the BHMF could only be expected if the local BHMF was substantially overestimated, and in this case larger duty cycles at low redshifts would be needed.

- Independently of the parameters of the model, we confirm an anti-hierarchical growth of SMBHs. Black holes of $M \gtrsim 10^{9} M_{\odot}$ stop forming at redshift 1 in all our models, while lower-mass SMBHs typically grow later or keep growing in all the redshift range according to the model. Antihierarchical behavior can also be observed in the duty cycle; at low redshift, this is close to 1 for objects of $M \lesssim 10^{7} M_{\odot}$ and decreases rapidly with the mass.

- Results for $\epsilon \geq 0.07$ and $\epsilon \sim 0.05$ imply quite different scenarios for the SMBH evolution at high redshifts. In the first case, a large number density of SMBHs, most of them quiescent, are already in place at $z \gtrsim 4$ (the mass density is approximately $10^{5} \mathrm{M}_{\odot} \mathrm{Mpc}^{-3}$ ). On the other hand, the model with $\epsilon=0.05$ predicts a small number density of SMBHs at high redshifts $\left(\rho_{\mathrm{BH}}<10^{4} M_{\odot} \mathrm{Mpc}^{-3} z \sim 4\right)$. The duty cycle 


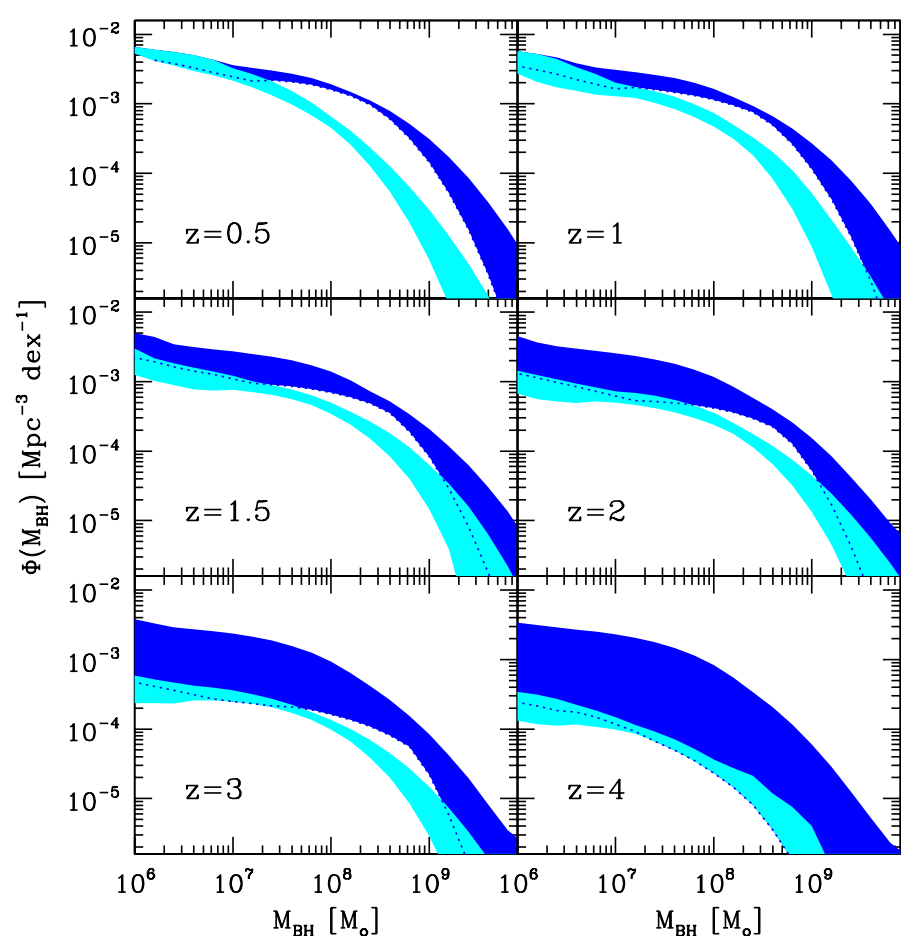

Fig. 15. Uncertainty on the predicted MF for SMBHs (blue shaded areas) and for AGN (cyan shaded areas) at different redshifts. This is obtained by combining the results and the related uncertainties from the models with $\epsilon=0.05,0.07$, and 0.1 . Dotted lines are the lower limits for the BHMF. We note the strong constraints imposed by the models on the evolution of AGN, compared to the large uncertainty in the BHMF at high redshifts.

is steadily increasing with redshift and most SMBHs are active at $z \simeq 4$.

- The evolution of active SMBHs also shows an antihierarchical behavior. The MF of low-mass AGN steadily increases with time, while for intermediate/high-mass AGN, we find a peak in the number density between redshift 1 and 2. As an example, the number density of $M \sim 10^{9} M_{\odot}$ AGN peaks around $z=1.5$ and it is approximately eight times larger than it is at present (and than at $z=3.4$, whose number density is equal to the local one).

- Information on the actual Eddington ratio distribution of SMBHs is still incomplete and uncertain. We have tested our results against different choices of the Eddington ratio distribution for type- 1 and type-2 AGN. In general, we observe a modest impact on the evolution of the AGN MF, compatible with the uncertainty of the model.

The main results of the paper can be summarized by Fig. 15. This provides a realistic estimate of the uncertainties on the SMBH and AGN mass function that arise from a continuity equation approach, on the basis of current knowledge (on, e.g., $\epsilon$, the local BHMF and QLF). These uncertainties are obtained by combining the results from the three best models discussed in the paper. We conclude that robust and strict predictions can be provided on the evolution of the mass function for type-1 and type- 2 AGN. The AGN MF is mainly determined by the QLF, with a small dependence on the choice of the radiative efficiency or of the local BHMF. The uncertainty only increases for very massive SMBHs, whose contribution to the observational QLF is small or negligible. On the other hand, we are less predictive of the evolution of the BHMF at high redshifts, which is very sensitive to model parameters.
This approach is complementary to other techniques that attempt to constrain the cosmic evolution of SMBHs and AGN, from analytical forward models to ab initio semi-analytical models and cosmological simulations. Our framework, once it has been calibrated to match the global properties of the observed population, as done in this paper, can be used as a starting point to investigate additional properties and make further predictions without tuning the set parameters.

The uncertainties in the (astro)physics of SMBHs and the lack of observational constraints, especially at high redshift and faint AGN luminosities, still allow relative freedom in choosing free parameters in analytical models and "sub-grid" physics. With the advent of future space missions, such as Athena and JSWT, dedicated to faint, high-redshift sources, we will be able to reduce the freedom in models, and advance our theoretical understanding.

Acknowledgements. M.T. thanks Martin Kunz for the helpful discussions on MCMC methods and their applications, and for providing the MCMC code used in this paper. M.T. also acknowledge support by the Swiss National Science Foundation. M.V. acknowledges funding from the European Research Council under the European Community's Seventh Framework Programme (FP7/20072013 Grant Agreement No. 614199, project "BLACK"). Part of the analysis was performed on the BAOBAB cluster at the University of Geneva.

\section{References}

Abel, T., Bryan, G. L., \& Norman, M. L. 2000, ApJ, 540, 39 Aird, J., Coil, A. L., Moustakas, J., et al. 2012, ApJ, 746, 90 Aversa, R., Lapi, A., de Zotti, G., Shankar, F., \& Danese, L. 2015, ApJ, 810, 74 Babić, A., Miller, L., Jarvis, M. J., et al. 2007, A\&A, 474, 755 Begelman, M. C., Volonteri, M., \& Rees, M. J. 2006, MNRAS, 370, 289 Berti, E., \& Volonteri, M. 2008, ApJ, 684, 822

Bongiorno, A., Zamorani, G., Gavignaud, I., et al. 2007, A\&A, 472, 443 Bongiorno, A., Merloni, A., Brusa, M., et al. 2012, MNRAS, 427, 3103 Booth, C. M., \& Schaye, J. 2009, MNRAS, 398, 53

Bower, R. G., Benson, A. J., Malbon, R., et al. 2006, MNRAS, 370, 645

Bromm, V., Coppi, P. S., \& Larson, R. B. 2002, ApJ, 564, 23

Cao, X. 2010, ApJ, 725, 388

Cao, X., \& Li, F. 2008, MNRAS, 390, 561

Caplar, N., Lilly, S., \& Trakhtenbrot, B. 2015, ApJ, 811, 148

Cavaliere, A., \& Vittorini, V. 2000, ApJ, 543, 599

Cavaliere, A., Morrison, P., \& Wood, K. 1971, ApJ, 170, 223

Cattaneo, A. 2001, MNRAS, 324, 128

Cattaneo, A., Blaizot, J., Devriendt, J., \& Guiderdoni, B. 2005, MNRAS, 364, 407

Croom, S. M., Richards, G. T., Shanks, T., et al. 2009, MNRAS, 399, 1755

Croton, D. J., Springel, V., White, S., et al. 2006, MNRAS, 365, 11

Davis, S. W., \& Laor, A. 2011, ApJ, 728, 98

Delvecchio, I., Gruppioni, C., Pozzi, F., et al. 2014, MNRAS, 439, 2736

Devecchi, B., \& Volonteri, M. 2009, ApJ, 694, 302

Di Matteo, T., Colberg, J., Springel, V., Hernquist, L., \& Sijacki, D. 2008, ApJ, 676,33

Dubois, Y., Devriendt, J., Slyz, A., \& Teyssier, R. 2010, MNRAS, 409, 985 Dubois, Y., Devriendt, J., Slyz, A., \& Teyssier, R. 2012, MNRAS, 420, 2662 Elvis, M., Risaliti, G., \& Zamorani, G. 2002, ApJ, 565, L75

Fanidakis, N., Baugh, C. M., Benson, A. J., et al. 2011, MNRAS, 410, 53 Fanidakis, N., Baugh, C. M., Benson, A. J., et al. 2012, MNRAS, 419, 2797 Fontanot, F., Pasquali, A., De Lucia, G., et al. 2011, MNRAS, 413, 957

Goulding, A. D., Alexander, D. M., Lehmer, B. D., \& Mullaney, J. R. 2010, MNRAS, 406, 597

Graham, A. W. 2007, MNRAS, 379, 711

Granato, G. L., Silva, L., Monaco, P., et al. 2001, MNRAS, 324, 757

Greene, J. E., \& Ho, L. C. 2007, ApJ, 667, 131

Haehnelt, M. G., \& Kauffmann, G. 2000, MNRAS, 318, L35

Haiman, Z., \& Loeb, A. 1998, ApJ, 503, 505

Haiman, Z., Quataert, E., \& Bower, G. C. 2004, ApJ, 612, 698

Häring, N., \& Rix, H.-W. 2004, ApJ, 604, L89

Hasinger, G. 2008, A\&A, 490, 905

Heckman, T. M., Kauffmann, G., Brinchmann, J., et al. 2004, ApJ, 613, 109

Hirschmann, M., Somerville, R. S., Naab, T., \& Burkert, A. 2012, MNRAS, 426, 237

Hirschmann, M., Dolag, K., Saro, A., et al. 2014, MNRAS, 442, 2304 
Hopkins, P. F., \& Hernquist, L. 2009, ApJ, 698, 1550

Hopkins, P. F., Narayan, R., \& Hernquist, L. 2006, ApJ, 643, 641

Hopkins, P. F., Richards, G. T., \& Hernquist, L. 2007, ApJ, 654, 731

Iwasawa, K., Gilli, R., Vignali, C., et al. 2012, A\&A, 546, A84

Kauffmann, G., \& Haehnelt, M. 2000, MNRAS, 311, 576

Kauffmann, G., \& Heckman, T. M. 2009, MNRAS, 397, 135

Kelly, B. C., \& Merloni, A. 2012, Adv. Astron., 2012, 970858

Kelly, B. C., \& Shen, Y. 2013, ApJ, 764, 45

Kelly, B. C., Vestergaard, M., \& Fan, X. 2009, ApJ, 692, 1388

Kormendy, J., \& Ho, L. C. 2013, ARA\&A, 51, 511

Kormendy, J., \& Richstone, D. 1995, ARA\&A, 33, 581

Koushiappas, S. M., Bullock, J. S., \& Dekel, A. 2004, MNRAS, 354, 292

La Franca, F., Fiore, F., Comastri, A., et al. 2005, ApJ, 635, 864

Li, Y.-R., Ho, L. C., \& Wang, J.-M. 2011, ApJ, 742, 33

Li, Y.-R., Wang, J.-M., \& Ho, L. C. 2012, ApJ, 749, 187

Lodato, G., \& Natarajan, P. 2007, MNRAS, 377, L64

Lusso, E., Comastri, A., Simmons, B. D., et al. 2012, MNRAS, 425, 623

Marconi, A., Risaliti, G., Gilli, R., et al. 2004, MNRAS, 351, 169

McConnell, N. J., \& Ma, C.-P. 2013, ApJ, 764, 184

Merloni, A., \& Heinz, S. 2008, MNRAS, 388, 1011

Merloni, A., Bongiorno, A., Brusa, M., et al. 2014, MNRAS, 437, 3550

Monaco, P., Salucci, P., \& Danese, L. 2000, MNRAS, 311, 279

Monaco, P., Fontanot, F., \& Taffoni, G. 2007, MNRAS, 375, 1189

Nobuta, K., Akiyama, M., Ueda, Y., et al. 2012, ApJ, 761, 143

Panessa, F., Bassani, L., Cappi, M., et al. 2006, A\&A, 455, 173

Raimundo, S. I., Fabian, A. C., Vasudevan, R. V., Gandhi, P., \& Wu, J. 2012, MNRAS, 419, 2529

Schulze, A., \& Wisotzki, L. 2010, A\&A, 516, A87

Schulze, A., Bongiorno, A., Gavignaud, I., et al. 2015, MNRAS, 447, 2085

Shakura, N. I., \& Sunyaev, R. A. 1973, A\&A, 24, 337

Shankar, F. 2013, Class. Quant. Grav., 30, 244001
Shankar, F., Weinberg, D. H., \& Miralda-Escudé, J. 2009, ApJ, 690, 20

Shankar, F., Sivakoff, G. R., Vestergaard, M., \& Dai, X. 2010, MNRAS, 401, 1869

Shankar, F., Weinberg, D. H., \& Miralda-Escudé, J. 2013, MNRAS, 428, 421

Shen, Y., \& Kelly, B. C. 2012, ApJ, 746, 169

Sijacki, D., Springel, V., di Matteo, T., \& Hernquist, L. 2007, MNRAS, 380, 877

Sijacki, D., Vogelsberger, M., Genel, S., et al. 2015, MNRAS, 452, 575

Small, T. A., \& Blandford, R. D. 1992, MNRAS, 259, 725

Soltan, A. 1982, MNRAS, 200, 115

Trump, J. R., Impey, C. D., Kelly, B. C., et al. 2011, ApJ, 733, 60

Ueda, Y., Akiyama, M., Ohta, K., \& Miyaji, T. 2003, ApJ, 598, 886

Ueda, Y., Akiyama, M., Hasinger, G., Miyaji, T., \& Watson, M. G. 2014, ApJ, 786, 104

Veale, M., White, M., \& Conroy, C. 2014, MNRAS, 445, 1144

Vestergaard, M., \& Osmer, P. S. 2009, ApJ, 699, 800

Vestergaard, M., Fan, X., Tremonti, C. A., Osmer, P. S., \& Richards, G. T. 2008, ApJ, 674, L1

Vika, M., Driver, S. P., Graham, A. W., \& Liske, J. 2009, MNRAS, 400, 1451

Volonteri, M. 2010, A\&ARv, 18, 279

Volonteri, M., Haardt, F., \& Madau, P. 2003, ApJ, 582, 559

Volonteri, M., Madau, P., Quataert, E., \& Rees, M. J. 2005, ApJ, 620, 69

Volonteri, M., Sikora, M., \& Lasota, J.-P. 2007, ApJ, 667, 704

Volonteri, M., Lodato, G., \& Natarajan, P. 2008, MNRAS, 383, 1079

Volonteri, M., Dubois, Y., Pichon, C., \& Devriendt, J. 2016, MNRAS, 460, 2979

Yu, Q., \& Tremaine, S. 2002, MNRAS, 335, 965

Yu, Q., Lu, Y., \& Kauffmann, G. 2005, ApJ, 634, 901

Wang, J.-M., Chen, Y.-M., \& Zhang, F. 2006, ApJ, 647, L17

Wang, J.-M., Hu, C., Li, Y.-R., et al. 2009, ApJ, 697, L141

Wu, S., Lu, Y., Zhang, F., \& Lu, Y. 2013, MNRAS, 436, 3271

Wyithe, J. S. B., \& Loeb, A. 2002, ApJ, 581, 886

Wyithe, J. S. B., \& Loeb, A. 2003, ApJ, 595, 614 


\section{Appendix A: Testing model assumptions}

Here we discuss solutions of the continuity equation with different values/combinations of the radiative efficiency and the local BHMF. Firstly, let us consider models with a local BHMF obtained by the convolution with 0.3 dex Gaussian scatter (LMF03). We only find acceptable fits to the observational QLF for $\epsilon \gtrsim 0.1$. The results for $\epsilon=0.1$ were discussed in the main part of the paper. When $\epsilon>0.1$, the BHMF does not evolve significantly over time for $M \lesssim 10^{8} M_{\odot}$, as shown by Fig. A.1. Instead, for $\epsilon<0.1$, the evolution of high-mass SMBHs is too fast and, consequently, the model strongly underestimates the high-luminosity part of the observational QLF at high redshifts because of the lack of active SMBHs with $M \gg 10^{8} M_{\odot}$. On the other hand, if the local mass function with 0.5 dex Gaussian scatter (LMF05) is used, we typically find better fits to the QLF independent of the choice of the radiative efficiency. Nevertheless, again, when $\epsilon \gtrsim 0.1$, the BHMF exhibits a smaller evolution over the whole range of masses (see Fig. A.1).

These results can be understood taking into account that the average accretion rate is proportional to $(1-\epsilon) / \epsilon$ and increases by a factor 2 reducing $\epsilon$ from 0.1 to 0.05 . If we use a local mass function with low number density of very massive SMBHs (as the LMF03 one), the duty cycle at these masses will be large in order to fit the high-luminosity tail of the QLF. The combination of a low radiative efficiency and a high duty cycle gives a high accretion rate and a fast evolution of high-mass SMBHs, whose number density will become too small at high redshifts. Viceversa, the argument can be overturned in the case of high radiative efficiencies associated to the LMF05 local mass function: the non-evolving MFs discussed above are then observed. We can conclude that, independently of the local BHMF, models with $\epsilon>0.1$ seem to be disfavored. They entail, in fact, an average accretion rate that is too low to produce SMBHs significantly growing with redshifts.

Figure A. 2 shows the $1 \sigma$ errors associated to the $\mathrm{SMBH}$ and AGN MFs for $\epsilon=0.07$, obtained from the MCMC method; they are relatively small, due to the strong constraints imposed by the quasar luminosity function. Clearly, they are not representative of the uncertainties of the model, that are mainly drawn by the uncertainties on the value of the average radiative efficiency and on the Eddington ratio distribution.

In Fig. A.2, we also investigate the effect of varying $\lambda_{\text {cut }}$, which defines the minimum accretion rate for an active SMBH. We extrapolate the power-law behavior of the Eddington ratio distribution for type- 2 AGN up to $\lambda_{\text {cut }}=10^{-5}$ (type- 1 AGN are not affected by the choice of $\left.\lambda_{\text {cut }}\right)$. This is probably an extreme situation because we expect a drop in $P(\lambda)$ somewhere below $\lambda \simeq 10^{-4}$ (Hopkins \& Hernquist 2009; Fanidakis et al. 2012). As expected, we find larger MFs, but the effect is modest both for SMBHs and for AGN.

Observations give only very partial constraints on the Eddington ratio distribution. For type-1 AGN they are limited to the highest values of the Eddington ratio $(\lambda \gtrsim 0.1)$, while for type-2 AGN the Eddington ratio distribution is determined over a large range of accretion rates (from $\sim 10^{-4}$ to 1 ) but only for $z<1$. Below, we discuss how the model predictions (especially in relation to the AGN MF) can change if different Eddington ratio distributions are employed. Results are shown in Fig. A.3.

- So far we have used a log-normal function to fit the Eddington ratio distributions estimated by Kelly \& Shen (2013). However, this is not the only possible choice. A truncated power law, as used for type-2 AGN (Eq. (10)), also provides a good fit to data. We use the same power-law index as

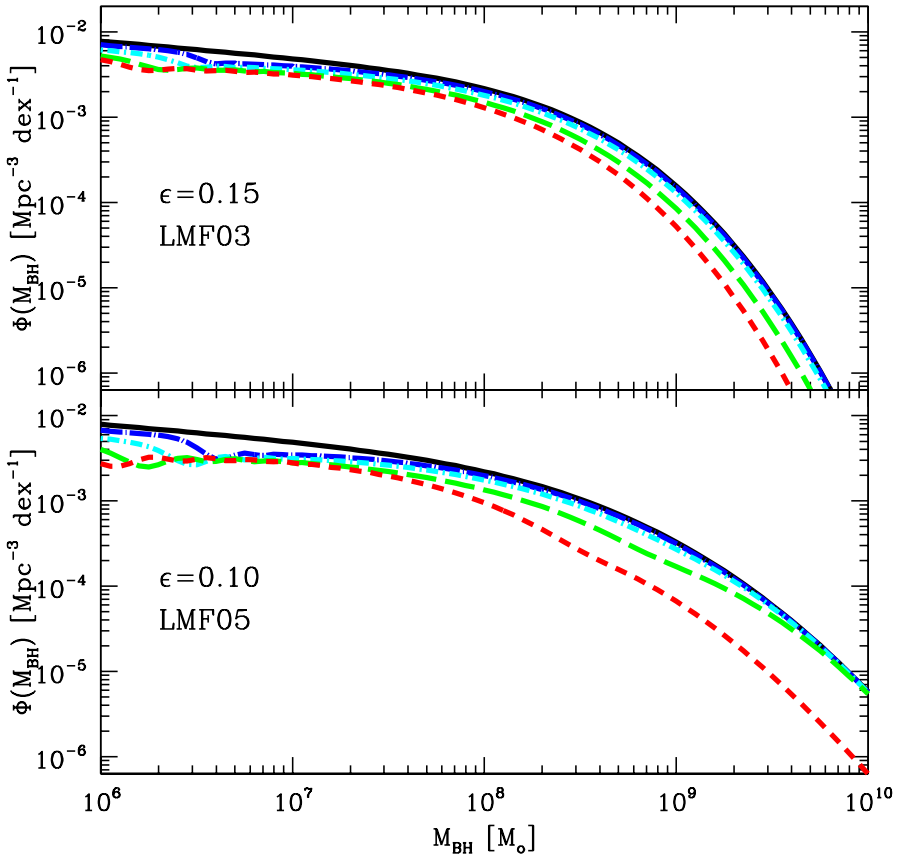

Fig. A.1. SMBH mass function obtained with $\epsilon=0.15$ and the LMF03 local BHMF (upper panel), and with $\epsilon=0.1$ and the LMF05 local BHMF (lower panel). The meaning of the lines are like in Fig. 9.

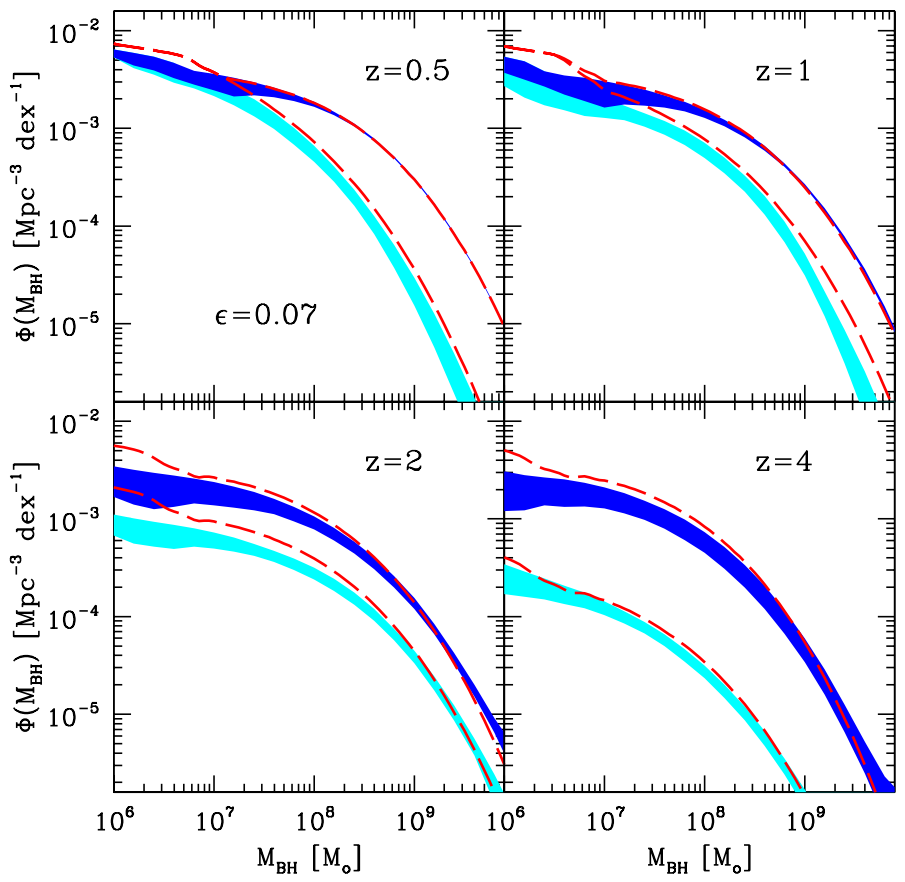

Fig. A.2. Uncertainties on the mass function of SMBHs (shaded blue areas) and of AGN (shaded cyan area) predicted by the model with $\epsilon=$ 0.07. Dashed red lines are the results using $\lambda_{\text {cut }}=10^{-5}$.

for type-2 AGN (Eq. (11)) and we find the cut-off $\lambda_{0}$ that best fits the Kelly \& Shen (2013) estimates at the different redshift bins. BHMFs are well compatible with previous results, while AGN MFs are typically larger, especially at high redshifts.

- The Eddington ratio distribution for type-2 AGN is determined only for $z<1$. We therefore test different redshift dependences of the power-law index with respect to the one 
used in Eq. (11). We consider two opposite situations: (1) we extrapolate the distribution observed at low redshifts to high redshifts, keeping a constant slope $\alpha_{\lambda}=-0.6$ over the whole redshift range; or (2) we use a steeper redshift dependence, $\alpha_{\lambda}=-0.6 /(0.4+z)^{1.5}$. As expected, the former gives mass functions that evolve more slowly at $z \gtrsim 1$. The effect is observed mainly at low/intermediate masses, at which the number density is larger by a factor $\$ 2$ with respect to the reference model. Finally, the second model does not produce significant changes in MFs.

We can conclude that using different Eddington ratio distributions seems to have a limited impact on the BHMF evolution. The uncertainty on this is dominated by the radiative efficiency, that can vary from $\epsilon=0.05$ to 0.1 . In principle, the AGN MF should be more sensitive to the choice of $P(\lambda)$. However, we see that the changes are not particularly relevant; they are only significantly larger than the intrinsic uncertainty associated to the models when we take a constant Eddington ratio distribution for type-2 AGN. The uncertainty on the Eddington ratio distribution then simply translates into a small increase of the uncertainty on the model predictions. This proves that our approach gives robust constraints on the AGN MF up to redshifts 4.
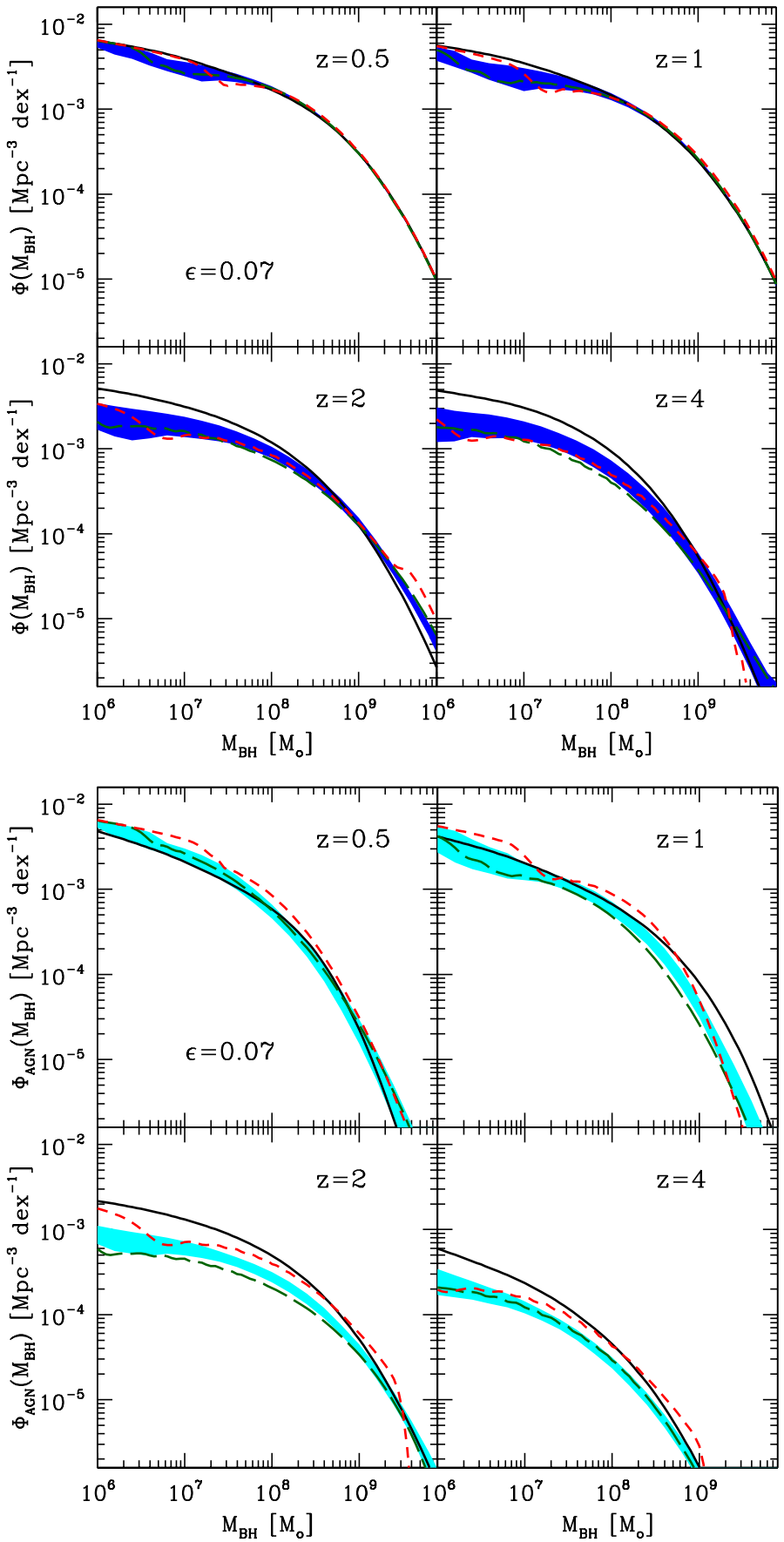

Fig. A.3. Mass function of SMBHs (top panel) and AGN (bottom panel) obtained after changing the Eddington ratio distribution. Lines are for following cases: (1) we use a truncated power law for $P(\lambda)$ of type-1 AGN (red short dashed lines); (2) a power-law distribution for type-2 AGN with constant slope $\alpha_{\lambda}=-0.6$ (black solid lines); (3) a faster evolving power-law distribution $\left(\alpha_{\lambda}=-0.6 /(0.4+z)^{1.5}\right)$ for type- 2 AGN (green long dashed lines). Shaded areas are for the uncertainty of the model as in Fig. A.2. 